TRANSACTIONS OF THE

AMERICAN MATHEMATICAL SOCIETY

Volume 365, Number 7, July 2013, Pages 3753-3774

S 0002-9947(2012)05745-X

Article electronically published on December 12, 2012

\title{
ON THE SMOOTHNESS OF CENTRALIZERS IN REDUCTIVE GROUPS
}

\author{
SEBASTIAN HERPEL
}

\begin{abstract}
Let $G$ be a connected reductive algebraic group over an algebraically closed field $k$. In a recent paper, Bate, Martin, Röhrle and Tange show that every (smooth) subgroup of $G$ is separable provided that the characteristic of $k$ is very good for $G$. Here separability of a subgroup means that its scheme-theoretic centralizer in $G$ is smooth. Serre suggested extending this result to arbitrary, possibly non-smooth, subgroup schemes of $G$. The aim of this paper is to prove this more general result. Moreover, we provide a condition on the characteristic of $k$ that is necessary and sufficient for the smoothness of all centralizers in $G$. We finally relate this condition to other standard hypotheses on connected reductive groups.
\end{abstract}

\section{INTRODUCTION}

Let $G$ be a connected reductive algebraic group over an algebraically closed field $k$. A closed subgroup $H \subseteq G$ is called separable in $G$ if the Lie algebra of its centralizer coincides with the infinitesimal fixed points of $H$ in $\operatorname{Lie}(G)$. Here, in contrast to the later sections of the paper, we do not consider scheme-theoretic centralizers. A similar notion exists for subalgebras of $\operatorname{Lie}(G)$. See BMR05 and BMRT for these concepts and their importance in the context of Serre's notion of $G$-complete reducibility. The starting point of this note is the following result of Bate, Martin, Röhrle and Tange ([BMRT, Thm. 1.2]). Liebeck-Seitz ([LS96, Thm. 3]) and Lawther-Testerman ([LT99, Thm. 2]) had earlier obtained special cases of this result.

Theorem. Suppose that char $k$ is very good for $G$. Then any subgroup of $G$ is separable in $G$ and any subalgebra of $\operatorname{Lie}(G)$ is separable in $\operatorname{Lie}(G)$.

Separability of a subgroup $H$ of $G$ can also be characterized as the smoothness of the scheme-theoretic centralizer of $H$ in $G$; see Section 3.1. In particular, all subgroups are separable if the characteristic of the ground field is zero (all algebraic group schemes in characteristic zero are smooth, due to a theorem of Cartier). The above theorem is therefore an instance of the general principle that a characteristic zero result becomes true in positive characteristic, provided that the characteristic is "big enough". More precisely, the assertion of the theorem can then be interpreted in the following way: If $H$ is an arbitrary closed subgroup scheme of $G$ and if char $k$ is very good for $G$, then the scheme-theoretic centralizer of $H$ in $G$ is smooth provided that either $H$ is smooth or $H$ is infinitesimal of height one (BMRT, Rem. 3.5(vi)]; see Lemma 3.1 below).

Received by the editors March 29, 2011 and, in revised form, November 4, 2011.

2010 Mathematics Subject Classification. Primary $20 \mathrm{G} 15$.

(C) 2012 American Mathematical Society Reverts to public domain 28 years from publication 
We are going to extend this result to arbitrary closed subgroup schemes, and to the weaker condition that the characteristic is only "pretty good for G" (Definition 2.11); see Theorem 3.3. We are also going to exhibit examples of non-smooth centralizers in small characteristic. In [BMRT Ex. 3.11], the authors construct non-separable subgroups of simple groups of types $G_{2}$ and $F_{4}$ in characteristic two. We observe that non-separable subgroups may be found in any reductive group in non-pretty good characteristic; see Example 4.2

Combining these results, the following is our main theorem:

Theorem 1.1. Let $G$ be a connected reductive algebraic group. Then the characteristic of $k$ is zero or pretty good for $G$ if and only if all centralizers of closed subgroup schemes in $G$ are smooth.

Finally, we show that the universal smoothness of centralizers holds in many classes of "standard" reductive groups, as introduced by Jantzen and McNinchTesterman; see Corollary 5.3. In fact, the different notions of standardness almost coincide (Theorem 5.2). This demonstrates that requiring the characteristic of the ground field to be pretty good for $G$ is a natural assumption for connected reductive groups.

The paper is organized as follows. In Section 2, we recall general facts about group schemes. We also recall the notions of good and very good primes. In Section 2.10, we introduce the notion of a pretty good prime.

In Section 3, we investigate conditions for the smoothness of centralizers. First, in Section 3.1, we relate the above notion of separability to the smoothness of certain scheme-theoretic centralizers. In Section [3.2, it is our goal to prove the forward implication of Theorem 1.1. In fact, we prove a slightly more general result in Theorem 3.3 .

In Section 4, we provide methods to construct non-smooth centralizers outside of pretty good characteristic. This allows us to give a proof of Theorem 1.1 at the end of this section.

Finally, in Section 5, we recall other notions of standard reductive groups, and prove that these amount to essentially the same as requiring the group to be defined in pretty good characteristic.

\section{Preliminaries}

In this section, let $k$ be a field and $\bar{k}$ a fixed algebraic closure of $k$. Let $S$ be a commutative ring.

2.1. Basic definitions. We call a functor from the category of $S$-algebras to the category of sets (resp. groups) an $S$-functor (resp. $S$-group functor). We adopt the point of view that (group) schemes over $S$ are certain $S$-(group) functors. See DG70] and [Ja03] for this approach. In particular, morphisms of (group) schemes are just morphisms of $S$-(group) functors. If $X$ is an $S$-(group)-functor and if $r: R \rightarrow R^{\prime}$ is a morphism of $S$-algebras, we write $x_{r}$ for the image of an element $x \in X(R)$ under the induced map $X(R) \rightarrow X\left(R^{\prime}\right)$. Since we are mainly interested in affine objects, let us recall the basic definitions: An affine $S$-(group) scheme is an $S$-(group) functor $X$ of the form $X=\operatorname{Hom}\left(A,{ }_{-}\right)$, where $A$ is an $S$-(Hopf) algebra (and where Hom denotes homomorphisms of $S$-algebras). We also write $S[X]$ instead of $A$. We say $X$ is of finite type or algebraic if $S[X]$ is a finitely 
generated $S$-algebra. If $R$ is an $S$-algebra, every affine $S$-(group) scheme $X$ gives rise to an affine $R$-(group) scheme $X_{R}$, with $R\left[X_{R}\right]=S[X] \otimes_{S} R$. If $M$ is an $S$ module, we define the $S$-group functor $M_{a}$ via $M_{a}(R)=M \otimes_{S} R$; see DG70, II, $\S 1$, 2.1]. If $M$ is projective and finite, then $M_{a}$ is an affine $S$-group scheme represented by the symmetric algebra $S\left(M^{*}\right)$.

2.2. Notions for group morphisms. Let $\varphi: G \rightarrow G^{\prime}$ be a morphism of affine algebraic $k$-group schemes. It is called surjective if the comorphism $\varphi^{*}: k\left[G^{\prime}\right] \rightarrow$ $k[G]$ is injective (which usually does not imply surjectivity of all induced maps $\left.G(R) \rightarrow G^{\prime}(R)\right)$. Surjectivity can be characterized by the following property (see Wat79, 15.5]): For every $k$-algebra $R$ and every $g^{\prime} \in G^{\prime}(R)$ there is a faithfully flat extension $s: R \rightarrow \bar{R}$ and an element $g \in G(\bar{R})$ such that $\varphi(\bar{R})(g)=g_{s}^{\prime}$.

Let $H \subseteq G$ and $H^{\prime} \subseteq G^{\prime}$ be closed subgroup schemes. The functor $\varphi^{-1} H^{\prime}: R \mapsto$ $\varphi(R)^{-1}\left(H^{\prime}(R)\right)$ is a closed subgroup scheme of $G$, defined by the ideal generated by $\varphi^{*}\left(I^{\prime}\right)$, if $I^{\prime} \subseteq k\left[G^{\prime}\right]$ defines $H^{\prime}$. The induced map $\varphi^{-1} H^{\prime} \rightarrow H^{\prime}$ is surjective if $\varphi$ is surjective (see [KMRT, VI, 22.4]). The kernel of $\varphi$ is the closed subgroup scheme $\operatorname{ker} \varphi:=\varphi^{-1}\{e\}$. Let $\varphi H$ be the closed subgroup scheme of $G^{\prime}$ defined by the ideal $\left(\varphi^{*}\right)^{-1}(I)$ if $I \subseteq k[G]$ defines $H$. Note that $\varphi(R)(H(R)) \subseteq(\varphi H)(R)$ for all $k$-algebras $R$. The induced map $H \rightarrow \varphi H$ is then surjective in the above sense, since its comorphism is injective. In particular,

$$
\begin{aligned}
(\varphi H)(R)=\left\{x \in G^{\prime}(R) \mid \text { there is a faithfully flat } s: R \rightarrow \bar{R}\right. \text { such that } \\
\left.\qquad x_{s} \in \varphi(\bar{R})(H(\bar{R}))\right\},
\end{aligned}
$$

where the forward containment follows from the above characterization of surjectivity. The reverse inclusion follows from the observation that $x_{s}\left(\varphi^{*}\right)^{-1}(I)=0$ implies $x\left(\varphi^{*}\right)^{-1}(I)=0$.

A sequence of morphisms of affine algebraic group schemes $1 \rightarrow N \stackrel{\varphi}{\rightarrow} G \stackrel{\psi}{\rightarrow}$ $H \rightarrow 1$ is called exact provided that $\psi$ is surjective and $\varphi$ induces an isomorphism of $N$ with $\operatorname{ker}(\psi)$. A morphism $\pi: G \rightarrow G^{\prime}$ of affine algebraic $k$-group schemes is called an isogeny if it is surjective and if its kernel is a finite group scheme.

2.3. Fixed points and centralizers. If $G$ is a $k$-group functor and $X$ is a $k$ functor, there is an obvious notion of a natural action $\alpha: G \times X \rightarrow X$. Suppose such an action is given. Then we can define the fixed point functor $X^{G}$. It associates to each $k$-algebra $R$ the set

$$
X^{G}(R)=\left\{x \in X(R) \mid \alpha\left(R^{\prime}\right)\left(g, x_{r}\right)=x_{r} \text { for every } r: R \rightarrow R^{\prime} \text { and } g \in G\left(R^{\prime}\right)\right\} .
$$

We also define the centralizer of a subfunctor $Y$ of $X$ in $G$ as the functor $\operatorname{Cent}_{G}(Y)$ that associates to each $k$-algebra $R$ the group

$$
\operatorname{Cent}_{G}(Y)(R)=\left\{g \in G(R) \mid \alpha\left(R^{\prime}\right)\left(g_{r}, y\right)=y \text { for every } r: R \rightarrow R^{\prime} \text { and } y \in Y\left(R^{\prime}\right)\right\} \text {. }
$$

It is well known that if $G, X$ and $Y$ are $k$-schemes and if $X$ is separated, then $X^{G}$ is closed in $X$, and $\operatorname{Cent}_{G}(Y)$ is closed in $G$ (see [Ja03, I, 2.6]).

We are interested in two special cases of the above situation. If $H$ is a closed subgroup scheme of an affine algebraic $k$-group scheme $G$, it acts on $G$ by conjugation. We denote the corresponding fixed point functor by $C_{G}(H)$. It is the same as 
the centralizer $\operatorname{Cent}_{G}(H)$, where $G$ acts on itself via conjugation. We call $C_{G}(H)$ the centralizer of $H$ in $G$. We have the equality

$$
C_{G}(H)_{\bar{k}}=C_{G_{\bar{k}}}\left(H_{\bar{k}}\right) \text {. }
$$

The center of $G$ is defined by $Z(G)=C_{G}(G)$. An isogeny $\pi: G \rightarrow G^{\prime}$ of affine algebraic $k$-group schemes is called central if $\operatorname{ker} \pi \subseteq Z(G)$.

If $M \subseteq N$ are two $k$-vector spaces, we can consider $M_{a}$ as a subfunctor of $N_{a}$. If $G$ acts linearly on $N_{a}$ (i.e. $R$-linearly on each $N \otimes R$ ), then $N$ is called a $G$-module. In this case, we write $\operatorname{Cent}_{G}(M)$ for the centralizer $\operatorname{Cent}_{G}\left(M_{a}\right)$.

2.4. Tangent spaces and Lie algebras. Let $X$ be an affine $S$-scheme. Let $S[\epsilon]=$ $S[T] / T^{2}$ with $\epsilon=\bar{T}$ denote the ring of dual numbers over $S$. Let $p: S[\epsilon] \rightarrow S$ be the map defined by $\epsilon \mapsto 0$. Let $x \in X(S)$. Then the (Zariski) tangent space of $X$ in $x$ is defined as a set by

$$
T_{x} X=\left\{y \in X(S[\epsilon]) \mid y_{p}=x\right\}
$$

see [DG70, II, $\S 4,3.3]$. Using $S[X]$, we can write this as

$$
\begin{aligned}
T_{x} X=\{x+g \epsilon \in X(S[\epsilon]) \mid & g: S[X] \rightarrow S \\
& \text { is an } S \text {-derivation with respect to } x: S[X] \rightarrow S\},
\end{aligned}
$$

and we can endow $T_{x} X$ with the $S$-module structure induced by the bijection with the space of all such point derivations. If $\varphi: X \rightarrow Y$ is a morphism of affine schemes that sends $x \in X(S)$ to $y \in Y(S)$, then it induces an $S$-linear map $d \varphi_{x}:=\left.\varphi(S[\epsilon])\right|_{T_{x} X}: T_{x} X \rightarrow T_{y} Y$.

If $G$ is an affine algebraic $S$-group scheme, we write $\operatorname{Lie}(G)=\mathfrak{g}=T_{e}(G)$, where $e \in G(S)$ is the identity element. Suppose that $\operatorname{char}(S)=p$. Then the $S$-module $\mathfrak{g}$ is finite and has the structure of a $p$-Lie algebra over $S$ (see [DG70, II, §7, 3.4]), and we call it the Lie algebra of $G$. This construction has the following converse (see Ibid., II, $§ 7,3.5$ ): If $\mathfrak{l}$ is a finite projective $S$-module with a $p$-Lie algebra structure over $S$, then there is an affine algebraic $S$-group scheme $\mathbb{G}(\mathfrak{l})$ with Lie algebra isomorphic to $\mathfrak{l}$ such that taking differentials yields a bijection

$$
\operatorname{Hom}(\mathbb{G}(\mathfrak{l}), G) \cong \operatorname{Hom}(\mathfrak{l}, \mathfrak{g}),
$$

where on the right-hand side we consider homomorphisms of $p$-Lie algebras over $S$. Moreover, $\mathbb{G}$ commutes with changing the base ring. If $S=k$ is a field, $\mathbb{G}$ induces an equivalence between the category of finite-dimensional $p$-Lie algebras and the category of algebraic $k$-group schemes of height one. Then for sub- $p$-Lie algebras $\mathfrak{l} \subseteq \operatorname{Lie}(G)$ (where $G$ is an affine algebraic $k$-group scheme), the group $\mathbb{G}(\mathfrak{l})$ can be identified with a closed subgroup scheme of $G$ (Ibid., II, $\S 7,4$ ).

If $G$ is an affine algebraic $k$-group scheme and if $S$ is a $k$-algebra, then $\operatorname{Lie}(G) \otimes$ $S \cong \operatorname{Lie}\left(G_{S}\right)$ (see Ibid., II, $\S 4,4.8$ ). In the same way we can prove part (a) of the following lemma. The other parts then follow from the definitions and an argument similar to Ibid., II, §4, 2.5.

Lemma 2.3. Let $X$ be an affine algebraic $k$-scheme. Let $x \in X(k)$. Let $G$ be an affine algebraic $k$-group scheme.

(a) The $k$-functors $R \mapsto T_{x_{R}} X_{R}$ and $\left(T_{x} X\right)_{a}$ are isomorphic. Here $x_{R}$ denotes the image of $x$ in $X(R)$.

(b) If $G$ acts naturally on $X$ and if $x \in X^{G}(k)$, then $G$ acts linearly on $T_{x} X$ and there is an equality $\left(T_{x} X\right)^{G}=T_{x}\left(X^{G}\right)$. 
(c) Let $\varphi: X \rightarrow Y$ be a $G$-equivariant morphism of affine algebraic $k$-schemes. Suppose that $x \in X^{G}(k)$. Then the induced linear map $d \varphi_{x}: T_{x} X \rightarrow$ $T_{\varphi(x)} Y$ is also G-equivariant.

If $G$ is an affine algebraic $k$-group scheme and if $g \in G(R)$, then conjugation with $g$ induces an automorphism $\operatorname{Int}(g): G_{R} \rightarrow G_{R}$, sending $h \in G\left(R^{\prime}\right)$ to $g_{r} h g_{r}^{-1}$ for each morphism $r: R \rightarrow R^{\prime}$ of $k$-algebras. This in turn induces an automorphism $\operatorname{Ad}(g)=d \operatorname{Int}(g): \operatorname{Lie}\left(G_{R}\right) \rightarrow \operatorname{Lie}\left(G_{R}\right)$. Using the above isomorphism of $k$-functors $\operatorname{Lie}\left(G_{\text {? }}\right) \cong \mathfrak{g}_{a}$, we see that $G$ acts linearly on $\mathfrak{g}_{a}$ via Ad. Now suppose that $H$ is a closed subgroup scheme of $G$, and that $i: H \rightarrow G$ is the inclusion. With the notation of Section 2.3 we find that

$$
\begin{aligned}
C_{G}(H)(R) & =\left\{g \in G(R) \mid \operatorname{Int}(g)=i_{R}: H_{R} \rightarrow G_{R}\right\}, \\
\operatorname{Cent}_{G}(\mathfrak{h})(R) & =\left\{g \in G(R) \mid \operatorname{Ad}(g)=d i_{R}: \operatorname{Lie}\left(H_{R}\right) \rightarrow \operatorname{Lie}\left(G_{R}\right)\right\} .
\end{aligned}
$$

Lemma 2.3 (b) also implies that

$$
\operatorname{Lie}\left(C_{G}(H)\right)=\mathfrak{g}^{H} .
$$

2.5. The identity component. Let $G$ be an affine algebraic $k$-group scheme. There is a finite group scheme $\pi_{0} G$ which is represented by the largest separable subalgebra of $k[G]$; see Wat79, Thm. 6.7]. Let $G^{\circ}$ denote the kernel of the corresponding morphism $G \rightarrow \pi_{0} G$. Then $G^{\circ}$ is a connected closed normal subgroup scheme of $G$, called the identity component of $G$.

Now let $G$ be an affine algebraic $S$-group scheme. For a prime ideal $\mathfrak{p} \subseteq S$, let $\kappa(\mathfrak{p})$ denote the residue field of the local ring $S_{\mathfrak{p}}$. Then we can construct the identity component of the affine $\kappa(\mathfrak{p})$-group scheme $G_{\kappa(\mathfrak{p})}$. For $x \in G(R)$ let $x_{\mathfrak{p}}$ denote the image of $x$ in $G_{\kappa(\mathfrak{p})}(R \otimes \kappa(\mathfrak{p}))$ under the obvious map. We define a subgroup functor $G^{\circ}$ of $G$ via

$$
G^{\circ}(R)=\left\{x \in G(R) \mid x_{\mathfrak{p}} \in G_{\kappa(\mathfrak{p})}^{\circ}(R \otimes \kappa(\mathfrak{p})) \text { for all prime ideals } \mathfrak{p} \subseteq S\right\},
$$

and we call $G^{\circ}$ the identity component of $G$. This construction commutes with base change. In particular, for an affine algebraic $k$-group scheme $G$ and a $k$-algebra $R$ we have

$$
\left(G^{\circ}\right)_{R}=\left(G_{R}\right)^{\circ}
$$

In fact, our definition coincides with the one in [SGA3, VI B, 3.1]. If $\varphi: G \rightarrow G^{\prime}$ is a morphism of affine algebraic $S$-group schemes, then it induces a morphism of $S$-group functors $G^{\circ} \rightarrow\left(G^{\prime}\right)^{\circ}$.

2.6. Smoothness and algebraic groups. Let $X$ be an affine algebraic $k$-scheme and consider a point $x \in X(k)$. Let $\operatorname{dim}_{x} X$ be the dimension of the local ring $k[X]_{x}$ obtained by localizing $k[X]$ at the kernel of $x$. We say that $x \in X(k)$ is a regular point if $\operatorname{dim}_{x} X=\operatorname{dim}_{k} T_{x} X$. This is equivalent to the regularity of $k[X]_{x}$. Using the dimension formula for flat morphisms (see DG70, I, §3, 6.3]), we get the following lemma.

Lemma 2.8. Let $\varphi: X \rightarrow Y$ be a flat morphism of affine algebraic $k$-schemes. Suppose that $x \in X(k)$ and $y=\varphi(x) \in Y(k)$ are regular points. Suppose that $x$ is also regular in the fibre $\varphi^{-1}(\{y\})$. Then the induced map $d \varphi_{x}: T_{x} X \rightarrow T_{y} Y$ is surjective (with kernel $T_{x}\left(\varphi^{-1}(\{y\})\right)$ ). 
An affine algebraic $k$-scheme $X$ is called smooth provided that $X_{\bar{k}}$ is regular, i.e. all prime ideals in $k[X] \otimes \bar{k}$ induce regular local rings. For affine algebraic $k$-group schemes, this property can be characterized in the following way (see [KMRT, 21.8, 21.9] and [DG70, II, §5, 2.1]):

Proposition 2.9. An affine algebraic k-group scheme $G$ is smooth if and only if any of the following equivalent statements hold:

(a) $G^{\circ}$ is smooth,

(b) the identity element $e \in G(k)$ is a regular point,

(c) $\operatorname{dim}_{k} \mathfrak{g}=\operatorname{dim} G$ (or, equivalently, $\operatorname{dim}_{k} \mathfrak{g} \leq \operatorname{dim} G$ ),

(d) $k[G] \otimes \bar{k}$ is reduced.

By a theorem of Cartier (see DG70, II, $\S 6,1.1]$ ), every affine algebraic $k$-group scheme $G$ is smooth if the characteristic of $k$ is zero. We reserve the term algebraic group over $k$ for a smooth affine algebraic $k$-group scheme. There is an equivalence of categories between the category of algebraic groups over $k$ and the category of linear algebraic groups over $\bar{k}$ that are equipped with a $k$-structure (see Bo91. and Sp98 for this language). This equivalence can be realized as the functor $G \mapsto G(\bar{k})$ if we regard $G(\bar{k})$ as an affine variety with coordinate $\operatorname{ring} k[G] \otimes \bar{k}$. It gives a correspondence between the smooth closed algebraic subgroups of $G$ and the closed subgroups of $G(\bar{k})$ that are defined over $k$. Moreover, the Lie algebras $\mathfrak{g} \otimes \bar{k}$ and the Lie algebra associated to $G(\bar{k})$ coincide.

If $1 \rightarrow N \rightarrow G \rightarrow H \rightarrow 1$ is an exact sequence of affine algebraic $k$-group schemes, we have that $\operatorname{dim} G=\operatorname{dim} N+\operatorname{dim} H$ and that $G$ is smooth if $H$ and $N$ are smooth (see [KMRT, 22.9-22.11]).

An isogeny $G \rightarrow G^{\prime}$ is called separable if its kernel is smooth. Two affine algebraic $k$-group schemes are called separably isogenous if there exists a separable isogeny between them.

2.7. Reductive groups. We say that an affine algebraic $k$-group scheme $G$ is a reductive algebraic group over $k$ if it is smooth and if $G(\bar{k})$ corresponds to a reductive linear algebraic group defined over $k$ via the equivalence given in Section 2.6. Equivalently, $G_{\bar{k}}$ has no non-trivial closed smooth connected normal unipotent subgroup schemes.

If $G$ is a reductive algebraic $\operatorname{group}$, we denote by $\operatorname{Rad}(G)$ its radical (i.e. the unique maximal torus of the center of $G$ ), and by $\operatorname{Der}(G)$ the derived group of $G$ (see [DG70, II, §5, 4.8]).

An affine algebraic $k$-group scheme $G$ is called linearly reductive provided that all $G$-modules are semisimple. This is equivalent to the vanishing of $H^{1}(G, V)$ for all finite-dimensional $G$-modules $V$ (see [DG70, II, §3, 3.7]).

Suppose $\left(G^{\prime}, G\right)$ is a pair of reductive algebraic groups such that $G \subseteq G^{\prime}$ is a closed subgroup. Then $\left(G^{\prime}, G\right)$ is called a reductive pair (see Richardson [Ri67]) provided that $\operatorname{Lie}\left(G^{\prime}\right)$ decomposes as a $G$-module (via the adjoint action restricted from $\left.G^{\prime}\right)$ into a direct sum $\operatorname{Lie}\left(G^{\prime}\right)=\operatorname{Lie}(G) \oplus \mathfrak{m}$.

2.8. Root data. A root datum $\mathcal{R}$ is a quadruple $\mathcal{R}=\left(X, \Phi, Y, \Phi^{\vee}\right)$, where $X$ and $Y$ are free abelian groups of finite rank that are in duality relative to a pairing $\langle\rangle:, X \times Y \rightarrow \mathbb{Z}$, and where $\Phi \subseteq X$ and $\Phi^{\vee} \subseteq Y$ are finite sets. The following additional axioms are required: (i) there is a bijection $\alpha \mapsto \alpha^{\vee}$ of $\Phi$ onto $\Phi^{\vee}$ 
such that $\left\langle\alpha, \alpha^{\vee}\right\rangle=2$; (ii) for each $\alpha \in \Phi$, the map $x \mapsto x-\left\langle x, \alpha^{\vee}\right\rangle \alpha$ (resp. $\left.y \mapsto y-\langle\alpha, y\rangle \alpha^{\vee}\right)$ stabilizes $\Phi$ (resp. $\left.\Phi^{\vee}\right)$. For a subset $\Phi^{\prime} \subseteq \Phi$, we write $\Phi^{\prime \vee}=$ $\left\{\alpha^{\vee} \mid \alpha \in \Phi^{\prime}\right\}$.

A root datum $\mathcal{R}$ is called reduced if $\alpha / 2 \notin \Phi$ for all $\alpha \in \Phi$. This means that the root systems $\Phi \subseteq \mathbb{R} \Phi$ and $\Phi^{\vee} \subseteq \mathbb{R} \Phi^{\vee}$ are reduced. A root datum is called semisimple provided that the ranks of $X$ and $\mathbb{Z} \Phi$ coincide. Suppose $\tilde{\mathcal{R}}=\left(\tilde{X}, \tilde{\Phi}, \tilde{Y}, \tilde{\Phi}^{\vee}\right)$ is another root datum, $f: X \rightarrow \tilde{X}$ is a $\mathbb{Z}$-linear map, and $f^{\vee}$ is the induced map $\tilde{Y} \rightarrow Y$. Then $f$ is called an isogeny of root data, and denoted $f: \mathcal{R} \rightarrow \tilde{\mathcal{R}}$, if $f$ is injective with finite cokernel, $f$ maps $\Phi$ onto $\tilde{\Phi}$ and $f^{\vee}$ maps $\tilde{\Phi}^{\vee}$ onto $\Phi^{\vee}$. See [SGA3, XXI] for more generalities on root data.

The significance of these notions is given by the following: Consider the category of pairs $(G, T)$, where $G$ is a connected reductive group over $\bar{k}$ and $T$ is a maximal torus in $G$, and where morphisms are central isogenies respecting the torus. Considering characters, roots, cocharacters and coroots relative to $T$, one associates a root datum $\mathcal{R}(G, T)$ to $(G, T)$. In this way one obtains a contravariant functor to the category of reduced root data with isogenies, which is almost an equivalence of categories (the functor is not faithful, but two central isogenies giving the same isogenies of root data differ only by conjugation with a torus element; to fix this one may add more data involving the choice of positive roots; see [SGA3, XXIII, Thm. 4.1]). The reductive group $G$ is semisimple if and only if its root datum is semisimple. Separable isogenies between connected reductive groups are automatically central and correspond to isogenies $f$ of root data such that $\operatorname{coker}(f)$ has order invertible in $k$. If $G$ has root datum $\mathcal{R}=\left(X, \Phi, Y, \Phi^{\vee}\right)$, then the dual root datum $\mathcal{R}^{\vee}=\left(Y, \Phi^{\vee}, X, \Phi\right)$ with the obvious pairing and bijection gives rise to the dual group $G^{\vee}$.

2.9. Good and very good primes. Let $\Phi$ be a (reduced) root system with irreducible components $\Phi_{1}, \ldots, \Phi_{t}$. Let $\Delta=\Delta_{1} \cup \cdots \cup \Delta_{t}$ be a base of $\Phi$, where each $\Delta_{i}$ is a base of $\Phi_{i}$. For each $i$, write the highest root $\tilde{\alpha}_{i}$ of $\Phi_{i}$ as a linear combination of simple roots. If a prime number $p$ divides any coefficient occurring among the expressions of the $\tilde{\alpha}_{i}$, it is said to be bad (for the root system $\Phi$ ). Otherwise it is called good. By inspecting the coefficients of the highest roots of irreducible root systems, one can verify that a prime $p$ is bad if and only if it occurs among the coefficients of the highest roots, if and only if it is smaller than the largest coefficient. A prime number $p$ is called very good for $\Phi$ if $p$ is good and if $p$ does not divide $n+1$ for any irreducible component of type $A_{n}$ in $\Phi$. Write $\Lambda=\left\{\lambda \in \mathbb{R} \Phi \mid\left\langle\lambda, \alpha^{\vee}\right\rangle \in \mathbb{Z}\right.$ for all $\left.\alpha \in \Phi\right\}$ for the weight lattice of $\Phi$. By inspecting the orders of the fundamental groups of the irreducible root systems one can verify that a prime $p$ is very good if and only if it is good and does not divide the order of the fundamental group of $\Phi$. The following more conceptual characterization of these notions is an easy generalization of [SpSt70, I, 4.1-4.5].

Lemma 2.10. Let $\Phi$ be a reduced root system with weight lattice $\Lambda$. Let $p$ be a prime number. Then the following hold:

(a) $p$ is good for $\Phi$ if and only if the $p$-torsion of $\mathbb{Z} \Phi / \mathbb{Z} \Phi^{\prime}$ vanishes for all subsets $\Phi^{\prime} \subseteq \Phi$,

(b) $p$ is very good for $\Phi$ if and only if the p-torsion of $\Lambda / \mathbb{Z} \Phi^{\prime}$ vanishes for all subsets $\Phi^{\prime} \subseteq \Phi$. 
Now let $G$ be a reductive algebraic group over $k$ and let $\Phi$ be the root system associated to $G_{\bar{k}}$. Then we call a prime number $p$ (very) good for $G$ provided that $p$ is (very) good for the root system $\Phi$.

2.10. Pretty good primes for root data. Let $\mathcal{R}=\left(X, \Phi, Y, \Phi^{\vee}\right)$ be a (reduced) root datum. Motivated by Lemma 2.10, we introduce the following terminology:

Definition 2.11. A prime number $p$ is called pretty good for the root datum $\mathcal{R}$ if the groups $X / \mathbb{Z} \Phi^{\prime}$ and $Y / \mathbb{Z} \Phi^{\prime \vee}$ have no $p$-torsion, for all subsets $\Phi^{\prime} \subseteq \Phi$. If $G$ is a connected reductive algebraic group over $k$, we say that a prime $p$ is pretty good for $G$ provided that it is pretty good for the root datum associated to $G_{\bar{k}}$.

This notion is related to the notions of good and very good primes for root systems as follows:

Lemma 2.12. Let $p$ be a prime number and $\mathcal{R}=\left(X, \Phi, Y, \Phi^{\vee}\right)$ as above. Then the following hold:

(a) $p$ is pretty good for $\mathcal{R}$ if and only if $p$ is good for $\Phi$ and the groups $X / \mathbb{Z} \Phi$ and $Y / \mathbb{Z} \Phi^{\vee}$ have no p-torsion;

(b) $p$ is very good for $\Phi \Rightarrow p$ is pretty good for $\mathcal{R} \Rightarrow p$ is good for $\Phi$;

(c) if $\mathcal{R}$ is semisimple, then $p$ is pretty good for $\mathcal{R}$ if and only if it is very good for $\Phi$;

(d) let $f: \mathcal{R} \rightarrow \tilde{\mathcal{R}}=\left(\tilde{X}, \tilde{\Phi}, \tilde{Y}, \tilde{\Phi}^{\vee}\right)$ be an isogeny of root data; if $\operatorname{coker}(f: X \rightarrow$ $\tilde{X})$ has no p-torsion, then $p$ is pretty good for $\mathcal{R}$ if and only if it is pretty good for $\tilde{\mathcal{R}}$;

(e) if $\mathcal{R}=\mathcal{R}_{1} \oplus \mathcal{R}_{2}$, then $p$ is pretty good for $\mathcal{R}$ if and only if it is so for both $\mathcal{R}_{1}$ and $\mathcal{R}_{2}$.

Proof. (a) For a subset $\Phi^{\prime} \subseteq \Phi$ we consider the exact sequence

$$
0 \rightarrow \mathbb{Z} \Phi / \mathbb{Z} \Phi^{\prime} \rightarrow X / \mathbb{Z} \Phi^{\prime} \rightarrow X / \mathbb{Z} \Phi \rightarrow 0 .
$$

If $p$ is pretty good, then the $p$-torsion of the middle group in this sequence vanishes, so $p$ is good by Lemma 2.10 (a). This proves the forward implication of (a). For the reverse implication, the above exact sequence and its obvious dual version imply that the $p$-torsion of $X / \mathbb{Z} \Phi^{\prime}$ (resp. $Y / \mathbb{Z} \Phi^{\prime \vee}$ ) coincide with the $p$-torsion of $\mathbb{Z} \Phi / \mathbb{Z} \Phi^{\prime}$ (resp. $\mathbb{Z} \Phi^{\vee} / \mathbb{Z} \Phi^{\prime \vee}$ ). Since $p$ is good for $\Phi$ and $\Phi^{\vee}$, the assertion again follows from Lemma 2.10 (a).

(b) According to (a) it remains to show: if $p$ is very good for $\Phi$, then $X / \mathbb{Z} \Phi$ and $Y / \mathbb{Z} \Phi^{\vee}$ have no $p$-torsion. For the first group, note that $(X \cap \mathbb{Q} \Phi) / \mathbb{Z} \Phi$ has no $p$-torsion according to Lemma 2.10 (b), since it is a subgroup of $\Lambda / \mathbb{Z} \Phi$. But then the same is true for $X / \mathbb{Z} \Phi$, since the quotient $X /(X \cap \mathbb{Q} \Phi)$ has no torsion at all. Since $p$ is also very good for $\Phi^{\vee}$, a similar argument proves the assertion for $Y / \mathbb{Z} \Phi^{\vee}$.

(c) Suppose that $\mathcal{R}$ is a semisimple root datum. According to (b) it remains to show that a pretty good prime $p$ for $\mathcal{R}$ does not divide the order of the fundamental group $\Lambda / \mathbb{Z} \Phi$. In the semisimple setting, the character lattice is a sublattice of the weight lattice, and the inclusion $X \rightarrow \Lambda$ is dual to the inclusion $\mathbb{Z} \Phi^{\vee} \rightarrow Y$. So both $\Lambda / X$ and $X / \mathbb{Z} \Phi$ have no $p$-torsion by assumption, and the assertion of (c) follows.

(d) The condition on $f$ guarantees that the $p$-torsion of $X / \mathbb{Z} \Phi^{\prime}$ coincides with the $p$-torsion of $\tilde{X} / \mathbb{Z} f\left(\Phi^{\prime}\right)$, and similarly for $f^{\vee}$, whose cokernel satisfies the same condition as the one of $f$. 
(e) Suppose $\mathcal{R}_{i}=\left(X_{i}, \Phi_{i}, Y_{i}, \Phi_{i}^{\vee}\right)$ for $i=1,2$. Then any subset $\Phi^{\prime} \subset \Phi=\Phi_{1} \cup \Phi_{2}$ decomposes as $\Phi^{\prime}=\Phi_{1}^{\prime} \cup \Phi_{2}^{\prime}$ with $\Phi_{i}^{\prime}=\Phi^{\prime} \cap \Phi_{i}$, and $X / \mathbb{Z} \Phi^{\prime} \cong X_{1} / \mathbb{Z} \Phi_{1}^{\prime} \oplus X_{2} / \mathbb{Z} \Phi_{2}^{\prime}$. A similar decomposition holds for $Y / \mathbb{Z} \Phi^{\prime \vee}$, which finishes the proof.

Example 2.13. The notion of a pretty good prime differs from the notions of good and very good primes. For instance, every prime is pretty good for $\mathrm{GL}_{2}$, whereas $p=2$ is not very good for $\mathrm{GL}_{2}$. On the other hand, all primes are good for $\mathrm{SL}_{2}$ and $\mathrm{PSL}_{2}$, whereas $p=2$ is not pretty good for $\mathrm{SL}_{2}$ or $\mathrm{PSL}_{2}$.

\section{Smooth CEnTralizers}

Let $k$ be a field and $\bar{k}$ an algebraic closure of $k$. Note that all constructions (preimages, centralizers, kernels, etc.) will be taken in the scheme-theoretic sense of Section 2 .

3.1. Relation to separability. Suppose for the moment that $G$ is an algebraic group over the algebraically closed field $\bar{k}$ of positive characteristic with Lie algebra $\mathfrak{g}$. This is the situation considered in BMRT]. There, a smooth closed subgroup $H \subseteq G$ is called separable in $G$ if $\operatorname{dim} C_{G(\bar{k})}(H(\bar{k}))=\operatorname{dim}_{\bar{k}} \mathfrak{g}^{H(\bar{k})}$, where we consider the centralizer to be taken in the category of linear algebraic groups. Similarly, a Lie subalgebra $\mathfrak{h} \subseteq \mathfrak{g}$ is called separable in $\mathfrak{g}$ if $\operatorname{dim}_{\operatorname{Cent}}(\bar{k})(\mathfrak{h})=\operatorname{dim}_{\bar{k}} \mathfrak{c}_{\mathfrak{g}}(\mathfrak{h})$. Again we consider here the centralizer as a linear algebraic group, whereas $\mathfrak{c}_{\mathfrak{g}}(\mathfrak{h})$ is the Lie algebra centralizer of $\mathfrak{h}$ in $\mathfrak{g}$.

The next lemma explains the assertion of [BMRT, Rem. 3.5(vi)], that relates the notion of separability to the smoothness of certain centralizers of subgroup schemes. We use the notation and equivalence of categories of Section 2.6.

Lemma 3.1. Let $G$ be an algebraic group over $\bar{k}$ with Lie algebra $\mathfrak{g}$. Let $H$ be a smooth subgroup scheme of $G$ and let $\mathfrak{h} \subseteq \mathfrak{g}$ be a subalgebra. Then the following hold:

(i) The centralizer $C_{G}(H)$ is smooth if and only if $H(\bar{k})$ is separable in $G(\bar{k})$.

(ii) Let $\mathfrak{h}^{\prime}$ be the p-envelope of $\mathfrak{h}$ in $\mathfrak{g}$, i.e. the smallest p-subalgebra of $\mathfrak{g}$ that contains $\mathfrak{h}$. Let $H^{\prime}=\mathbb{G}\left(\mathfrak{h}^{\prime}\right)$ be the affine group scheme associated to $\mathfrak{h}^{\prime}$ as in Section 2.4. Then $C_{G}\left(H^{\prime}\right)$ is smooth if and only if $\mathfrak{h}$ is separable in $\mathfrak{g}$.

Proof. (i) Let $i: H \rightarrow G$ be the inclusion. The smoothness of $H$ implies that for $g \in G(\bar{k})$, we have $\operatorname{Int}(g)=i: H \rightarrow G$ if and only if $\operatorname{Int}(g)(\bar{k})=i(\bar{k})$ (see [Liu02, Ex. 2.9 in 3.2]). Therefore, according to (2.4), we get the identity

$$
C_{G}(H)(\bar{k})=C_{G(\bar{k})}(H(\bar{k})) .
$$

This implies that the dimensions of the scheme $C_{G}(H)$ and the linear algebraic group $C_{G(\bar{k})}(H(\bar{k}))$ coincide (because the defining ideal of the linear algebraic group now proves to be the radical of the ideal defining $\left.C_{G}(H)\right)$. By (2.6), we also have that $\operatorname{Lie}\left(C_{G}(H)\right)=\mathfrak{g}^{H}=\mathfrak{g}^{H(\bar{k})}$ (for the last equation we note that both subsets of $\mathfrak{g}$ can be defined in terms of the same comodule map). We conclude by Proposition 2.9 that $C_{G}(H)$ is smooth if and only if $\operatorname{dim} C_{G}(H)=\operatorname{dim}_{\bar{k}} \mathfrak{g}^{H(\bar{k})}$, if and only if $\operatorname{dim} C_{G(\bar{k})}(H(\bar{k}))=\operatorname{dim}_{\bar{k}} \mathfrak{g}^{H(\bar{k})}$. The last equality is equivalent to the separability of $H(\bar{k})$ in $G(\bar{k})$.

(ii) As in part (i) we first get the identity $\operatorname{Cent}_{G}(\mathfrak{h})(\bar{k})=\operatorname{Cent}_{G(\bar{k})}(\mathfrak{h})$. We also have the identity $\operatorname{Lie}\left(\operatorname{Cent}_{G}(\mathfrak{h})\right)=\mathfrak{c}_{\mathfrak{g}}(\mathfrak{h})$ and can therefore conclude in the same 
manner as in part (i) that $\mathfrak{h}$ is separable in $\mathfrak{g}$ if and only if $\operatorname{Cent}_{G}(\mathfrak{h})$ is smooth. Since the actions of $G(\bar{k})$ and $\mathfrak{g}$ on $\mathfrak{g}$ are compatible with the $p$-mapping, we get that $\mathfrak{h}$ is separable in $\mathfrak{g}$ if and only $\mathfrak{h}^{\prime}$ is separable in $\mathfrak{g}$ (in fact, $\mathfrak{c}_{\mathfrak{g}}(\mathfrak{h})=\mathfrak{c}_{\mathfrak{g}}\left(\mathfrak{h}^{\prime}\right)$ and $\left.\operatorname{Cent}_{G(\bar{k})}(\mathfrak{h})=\operatorname{Cent}_{G(\bar{k})}\left(\mathfrak{h}^{\prime}\right)\right)$. It now suffices to show that $\operatorname{Cent}_{G}\left(\mathfrak{h}^{\prime}\right)=C_{G}\left(H^{\prime}\right)$. But for $g \in G(R)$ we have that $\operatorname{Ad}(g)=d i_{R}: \operatorname{Lie}\left(H_{R}^{\prime}\right) \rightarrow \operatorname{Lie}\left(G_{R}\right)$ if and only if $\operatorname{Int}(g)=i_{R}: H_{R}^{\prime} \rightarrow G_{R}$ (see (2.2) ). So the desired equality follows from the identities (2.4) and (2.5)

3.2. Sufficient conditions for the smoothness of centralizers. A sufficient condition for the smoothness of fixed points is given by the following theorem (see DG70, II, §5, 2.8]):

Theorem 3.2. Let $G$ and $H$ be affine algebraic $k$-group schemes. Suppose that $G$ is smooth and that $H$ acts via group automorphisms on $G$. If $H^{1}(H, \mathfrak{g})=0$ for the corresponding $H$-module structure on $\mathfrak{g}$, then $G^{H}$ is a smooth affine algebraic group scheme. In particular, $G^{H}$ is smooth if $H$ is linearly reductive.

Our goal is to prove the following theorem:

Theorem 3.3. Let $G$ be a reductive algebraic group over $k$. Suppose that the characteristic of $k$ is zero or pretty good for $G^{\circ}$. Suppose further that the component group $\pi_{0} G(\bar{k})$ has order invertible in $k$. Then for any closed subgroup scheme $H \subseteq$ $G$, the centralizer $C_{G}(H)$ is smooth. In particular, all centralizers of closed subgroup schemes are smooth for a connected reductive group $G$ in pretty good characteristic.

We defer the proof of the theorem to the end of this section. It relies on a series of lemmas which generalize ideas of [BMRT] to the scheme-theoretic setup. The main steps of the proof then closely follow the proof of [BMRT, Thm. 1.2], plus a careful type $A$ analysis.

Remarks 3.4. (a) Theorem 3.3 in particular answers a question raised in BMRT, Rem. 3.5(vi)], attributed to Serre: Even the centralizers of non-smooth subgroup schemes of a connected reductive group $G$ are smooth in very good characteristic.

(b) The cohomology criterion of Theorem 3.2 does not apply in the situation of Theorem 3.3. For example, take $k$ to be a field of characteristic 3. Let $H$ be the constant $k$-group scheme $\mathbb{Z} / 3 \mathbb{Z}$, realized as a closed subgroup scheme of $\mathrm{GL}_{2}$ via

$$
H(R)=\left\{\left(\begin{array}{ll}
1 & r \\
0 & 1
\end{array}\right) \mid r^{3}=r\right\}
$$

for each $k$-algebra $R$. Then $H^{1}\left(H, \mathfrak{g l}_{2}\right)$ is one-dimensional as a $k$-vector space (which can be checked by computing the cohomology of the cyclic group of order 3 with the corresponding action on $k^{4}$ ). So the cohomology criterion does not apply for $\mathrm{GL}_{2}$, whereas all primes are pretty good for $\mathrm{GL}_{2}$.

(c) As pointed out to the author by Steve Donkin, there is the following more generic way to produce examples of non-vanishing cohomology on some $\mathfrak{g l}_{n}$ as above. Take any affine algebraic group scheme $G$ that is not linearly reductive. Then there exist $G$-modules $M_{1}, M_{2}$ such that $\operatorname{Ext}_{G}^{1}\left(M_{1}, M_{2}\right)$ is not trivial. Setting $V=M_{1} \oplus M_{2}$, we find that $H^{1}(G, \mathfrak{g l}(V))=H^{1}\left(G, V \otimes V^{*}\right) \supseteq H^{1}\left(G, M_{2} \otimes M_{1}^{*}\right) \cong$ $\operatorname{Ext}_{G}^{1}\left(k, M_{2} \otimes M_{1}^{*}\right) \cong \operatorname{Ext}_{G}^{1}\left(M_{1}, M_{2}\right) \neq 0$. If $V$ also happens to be a faithful $G$ module (this can be arranged for example for any simple adjoint algebraic group $G$ in positive characteristic), then we can identify $G$ with its image in GL( $V)$. This 
again yields an example where the theorem implies the smoothness of the centralizer $C_{\mathrm{GL}(V)}(G)$, whereas the cohomology criterion does not apply.

(d) The following example (BMRT, Rem. 3.5]) shows that the assertion of the theorem may fail for non-connected reductive groups without the hypothesis on the component group. Take $G=\mathbb{G}_{m} \rtimes \operatorname{Aut}\left(\mathbb{G}_{m}\right)=\mathbb{G}_{m} \rtimes \mathbb{Z} / 2 \mathbb{Z}$. Then $C_{G}(\mathbb{Z} / 2 \mathbb{Z})=$ $\mu_{2} \rtimes \mathbb{Z} / 2 \mathbb{Z}$ is smooth if and only if $\operatorname{char}(k) \neq 2$.

The following three lemmas are used in the proof of Theorem 3.3 . The key idea is to transfer the desired property from $\mathrm{GL}_{n}$, where all centralizers are smooth, according to the following first lemma.

Lemma 3.5. Let $H$ be a closed subgroup scheme of $\mathrm{GL}_{n}$. Then the centralizer $C_{\mathrm{GL}_{n}}(H)$ is smooth.

Proof. According to (2.1) and Proposition 2.9 we can assume that $k=\bar{k}$ is algebraically closed. We have the identity $\left(\mathfrak{g l}_{n}^{H}\right)_{a}=\left(\left(\mathfrak{g l}_{n}\right)_{a}\right)^{H}$ (see DG70, II, §2, 1.6]). In particular, $\left(\left(\mathfrak{g l}_{n}\right)_{a}\right)^{H}$ can be represented by a reduced $k$-algebra (see Section 2.1). Let $R$ be a $k$-algebra and let us identify $\mathfrak{g l}_{n} \otimes R=M_{n \times n}(R)$, where the right-hand side denotes $n \times n$-matrices with coefficients in $R$. We can write

$$
\begin{aligned}
\left(\left(\mathfrak{g l}_{n}\right)_{a}\right)^{H}(R) & =\left\{x \in M_{n \times n}(R) \mid \operatorname{Ad}(h)\left(x_{r}\right)=x_{r} \text { for every } r: R \rightarrow R^{\prime} \text { and } h \in H\left(R^{\prime}\right)\right\} \\
& =\left\{x \in M_{n \times n}(R) \mid h x_{r} h^{-1}=x_{r} \text { for every } r: R \rightarrow R^{\prime} \text { and } h \in H\left(R^{\prime}\right)\right\}, \\
C_{\mathrm{GL}_{n}}(H)(R) & =\left\{g \in \mathrm{GL}_{n}(R) \mid h g_{r} h^{-1}=g_{r} \text { for every } r: R \rightarrow R^{\prime} \text { and } h \in H\left(R^{\prime}\right)\right\} \\
& =\left\{x \in\left(\left(\mathfrak{g l}_{n}\right)_{a}\right)^{H}(R) \mid \operatorname{det}(x) \text { is invertible }\right\} .
\end{aligned}
$$

So $C_{\mathrm{GL}_{n}}(H)$ can be represented by the localization $A_{\text {det }}$ if $A$ represents $\left(\left(\mathfrak{g l}_{n}\right)_{a}\right)^{H}$. Since $A$ is reduced, the same is true for $A_{\text {det }}$. Hence $C_{\mathrm{GL}_{n}}(H)$ is smooth (Proposition 2.9).

The next lemma shows how the smoothness of centralizers descends from one reductive group to the other, provided that the inclusion induces a reductive pair (see Section 2).

Lemma 3.6. Let $\left(G^{\prime}, G\right)$ be a reductive pair of reductive algebraic groups over $k$ and let $H \subseteq G$ be a closed subgroup scheme. If $C_{G^{\prime}}(H)$ is smooth, then so is $C_{G}(H)$.

Proof. We proceed in three steps. Let $\mathfrak{g}^{\prime}=\mathfrak{g} \oplus \mathfrak{m}$ be an $H$-module decomposition of $\mathfrak{g}^{\prime}$.

1. It suffices to show that $c:=\operatorname{dim} C_{G^{\prime}}(H)-\operatorname{dim} C_{G}(H) \leq \operatorname{dim}_{k} \mathfrak{m}^{H}$. Indeed, by assumption (and using (2.6) ) we know that $\operatorname{dim} C_{G^{\prime}}(H)=\operatorname{dim}_{k} \mathfrak{g}^{\prime H}=\operatorname{dim}_{k} \mathfrak{g}^{H}+$ $\operatorname{dim}_{k} \mathfrak{m}^{H}$. So the desired inequality $\operatorname{dim} C_{G}(H) \geq \operatorname{dim}_{k} \mathfrak{g}^{H}$ (see Proposition 2.9) is equivalent to $c \leq \operatorname{dim}_{k} \mathfrak{m}^{H}$.

2. We show that $c \leq \operatorname{dim}_{k} T_{\bar{e}}\left(\left(G^{\prime} / G\right)^{H}\right)$. Consider the quotient $X:=C_{G^{\prime}}(H) /$ $C_{G}(H)$. It is a smooth algebraic scheme of dimension $\operatorname{dim} X=\operatorname{dim}_{\bar{e}} X=c$ (see [DG70, III, $\S 32.7,5.4$ and $5.5(\mathrm{a})]$ ), which can be formally obtained as the faisceau associated to the functor

$$
F_{1}: R \mapsto C_{G^{\prime}}(H)(R) / C_{G}(H)(R) ;
$$

see [Ja03, I, 5.6]. Now if we start with the functor $F_{2}: R \mapsto G^{\prime}(R) / G(R)$ we get as the associated faisceau the smooth algebraic scheme $G^{\prime} / G$ (which is in fact affine, due to the reductivity assumptions; see [Ri77]). Moreover, we have an inclusion of 
functors $F_{2} \hookrightarrow G^{\prime} / G$ ([Ja03, I, 5.6]). There is an obvious action of $H$ on $F_{2}$ by conjugation, which extends uniquely to an action of $H$ on $G^{\prime} / G$ (see DG70. III, $\S 3,1.3])$. We now have $F_{1} \hookrightarrow F_{2}^{H} \hookrightarrow\left(G^{\prime} / G\right)^{H}$, where the first inclusion follows from the fact that $C_{G^{\prime}}(H) \cap G=C_{G}(H)$. Therefore, we have a monomorphism $X \hookrightarrow\left(G^{\prime} / G\right)^{H}$ (see [Ja03, I, $\left.5.4(4)\right]$ ). Note that $\left(G^{\prime} / G\right)^{H}$ is closed in $G^{\prime} / G$ (Ibid., I, $2.6(10))$ and hence also affine. We conclude that $c=\operatorname{dim}_{\bar{e}} X \leq \operatorname{dim}_{\bar{e}}\left(G^{\prime} / G\right)^{H} \leq$ $\operatorname{dim}_{k} T_{\bar{e}}\left(\left(G^{\prime} / G\right)^{H}\right)$.

3. We show that $T_{\bar{e}}\left(G^{\prime} / G\right)^{H} \cong \mathfrak{m}^{H}$ as $k$-vector spaces. Let us first note that the natural map $\pi: G^{\prime} \rightarrow G^{\prime} / G$ is $H$-equivariant with respect to the action defined in step 2 (because $\pi$ is the composite $G^{\prime} \rightarrow F_{2} \rightarrow G^{\prime} / G$ ). It is also flat with smooth fibre $\pi^{-1}(\bar{e})=G$ (see DG70, III, $\left.\S 3,2.5\right]$ ), and therefore the induced map $d \pi_{e}: \mathfrak{g}^{\prime} \rightarrow T_{\bar{e}}\left(G^{\prime} / G\right)$ is $H$-equivariant and surjective, with kernel $\mathfrak{g}$, according to Lemmas 2.3 (c) and 2.8. So now $\mathfrak{m} \cong T_{\bar{e}}\left(G^{\prime} / G\right)$ as $H$-modules, so that $\mathfrak{m}^{H} \cong$ $\left(T_{\bar{e}}\left(G^{\prime} / G\right)\right)^{H}=T_{\bar{e}}\left(\left(G^{\prime} / G\right)^{H}\right)$, where the last equality is Lemma 2.3 (b).

Remark 3.7. Let $H \subseteq G$ be a closed subgroup scheme of a smooth affine algebraic $k$ group scheme and suppose that $H^{1}(H, \mathfrak{g})=0$. Then $C_{G}(H)$ is smooth, according to Theorem 3.2. We can also use the above two lemmas to obtain a new proof for this fact: Pick a closed embedding $G \hookrightarrow \mathrm{GL}_{n}$ for a suitable $n$ (see Wat79, 3.4]). By Lemma 3.5. $C_{\mathrm{GL}_{n}}(H)$ is smooth. If $H^{1}(H, \mathfrak{g})=0$, then $\operatorname{dim}_{k} \mathfrak{g l}_{n}^{H}=$ $\operatorname{dim}_{k} \mathfrak{g}^{H}+\operatorname{dim}_{k}\left(\mathfrak{g l}_{n} / \mathfrak{g}\right)^{H}$. Now the three steps of the proof of Lemma 3.6 go through with $G^{\prime}=\mathrm{GL}_{n}$ and $\mathfrak{m}$ replaced by $\mathfrak{g l}_{n} / \mathfrak{g}$ (we also need to extend our tangent space arguments to the possibly non-affine variety $\left.G^{\prime} / G\right)$.

The following final lemma states that the smoothness of a centralizer of a subgroup scheme depends on the ambient group only up to central, separable isogenies.

Lemma 3.8. Let $\pi: G \rightarrow G^{\prime}$ be a central, separable isogeny of smooth affine algebraic $k$-group schemes, and suppose that $H \subseteq G$ is a closed subgroup scheme. Then $C_{G}(H)$ is smooth if and only if $C_{G^{\prime}}(\pi H)$ is smooth. In particular, all centralizers in $G$ are smooth if and only if the same is true for $G^{\prime}$.

Proof. We let $H$ act on $G^{\prime}$ via $h . x=\pi(h) x \pi(h)^{-1}$ for $h \in H(R), x \in G^{\prime}(R)$. In this way $\pi: G \rightarrow G^{\prime}$ becomes an $H$-equivariant morphism. Since ker $\pi$ is smooth and finite, the differential $d \pi: \mathfrak{g} \rightarrow \mathfrak{g}^{\prime}$ is injective. Moreover, according to our smoothness assumptions and Lemmas 2.3 (c) and 2.8, it is also $H$-equivariant and surjective. Thus it induces an isomorphism $\mathfrak{g}^{H} \cong \mathfrak{g}^{\prime H}$.

We claim that $\left(G^{\prime}\right)^{H}=C_{G^{\prime}}(\pi H)$. The reverse inclusion follows from the definitions. Conversely, suppose that $x \in\left(G^{\prime}\right)^{H}(R)$. Let $r: R \rightarrow R^{\prime}$ be a $k$-algebra homomorphism and let $h^{\prime} \in \pi H\left(R^{\prime}\right)$. We have to show that $h^{\prime} x_{r} h^{\prime-1}=x_{r}$. There is a faithfully flat extension $s: R^{\prime} \rightarrow S$ and an element $h \in H(S)$ such that $h_{s}^{\prime}=\pi(h)$. By our choice of $x$ we get that $x_{s \circ r}=h . x_{s \circ r}=h_{s}^{\prime} x_{s \circ r} h_{s}^{\prime-1}$. Now the injectivity of $s$ implies that $x_{r}=h^{\prime} x_{r} h^{\prime-1}$, as required.

We now have established that $\operatorname{Lie}\left(C_{G}(H)\right)=\mathfrak{g}^{H} \cong \mathfrak{g}^{\prime H}=\operatorname{Lie}\left(C_{G^{\prime}}(\pi H)\right)$. To prove the assertion, it therefore remains to show that $\operatorname{dim} C_{G}(H)=\operatorname{dim} C_{G^{\prime}}(\pi H)$. Let $M=\pi^{-1} C_{G^{\prime}}(\pi H) \subseteq G$. The map $\pi$ induces an exact sequence

$$
1 \rightarrow \operatorname{ker}(\pi) \rightarrow M \rightarrow C_{G^{\prime}}(\pi H) \rightarrow 1 .
$$

It follows that $\operatorname{dim} M=\operatorname{dim} C_{G^{\prime}}(\pi H)$. By construction, we have $C_{G}(H) \subseteq \pi^{-1}\left(G^{\prime}\right)^{H}$ $=M$. To show that $\operatorname{dim} C_{G}(H)=\operatorname{dim} C_{G^{\prime}}(\pi H)$, it therefore suffices to check that $M^{\circ} \subseteq C_{G}(H)^{\circ}$. 
Let $m \in M^{\circ}(R)$ and $h \in H\left(R^{\prime}\right)$ for some morphism of $k$-algebras $r: R \rightarrow R^{\prime}$. We need to show that $h m_{r} h^{-1} m_{r}^{-1}=e_{R^{\prime}}$. Consider the natural map of $R^{\prime}$-functors

$$
\begin{gathered}
\varphi: M_{R^{\prime}}^{\circ} \rightarrow(\operatorname{ker} \pi)_{R^{\prime}}, \\
M^{\circ}\left(R^{\prime \prime}\right) \ni x \mapsto h_{s} x h_{s}^{-1} x^{-1} \text { for } s: R^{\prime} \rightarrow R^{\prime \prime} .
\end{gathered}
$$

We first note that $\varphi$ is well defined. Indeed, if $x \in M^{\circ}\left(R^{\prime \prime}\right)$, then $\pi(x)$ commutes with all elements of $\pi H\left(R^{\prime \prime}\right)$. In particular, it commutes with $\pi\left(h_{s}\right)$. But then $h_{s} x h_{s}^{-1} x^{-1}$ has to lie in $\operatorname{ker} \pi\left(R^{\prime \prime}\right)$. In fact, $\varphi$ is even a morphism of affine group schemes over $R^{\prime}$, because we can compute that

$$
\begin{aligned}
\varphi\left(x x^{\prime}\right) & =h_{s} x x^{\prime} h_{s}^{-1} x^{-1} x^{-1}=h_{s} x h_{s}^{-1} h_{s} x^{\prime} h_{s}^{-1} x^{-1} x^{-1} \\
& =h_{s} x h_{s}^{-1} \varphi\left(x^{\prime}\right) x^{-1}=\varphi(x) \varphi\left(x^{\prime}\right),
\end{aligned}
$$

where for the last equation we use that $\operatorname{ker}(\pi) \subseteq Z(G)$. Taking identity components, we get an induced homomorphism $\varphi^{\circ}:\left(M_{R^{\prime}}^{\circ}\right)^{\circ} \rightarrow\left((\operatorname{ker} \pi)_{R^{\prime}}\right)^{\circ}$. Now, according to (2.7), $\left(M_{R^{\prime}}^{\circ}\right)^{\circ}=\left(\left(M^{\circ}\right)^{\circ}\right)_{R^{\prime}}=\left(M^{\circ}\right)_{R^{\prime}}$ and $\left((\operatorname{ker} \pi)_{R^{\prime}}\right)^{\circ}=\left((\operatorname{ker} \pi)^{\circ}\right)_{R^{\prime}}=\{e\}_{R^{\prime}}=$ $\left\{e_{R^{\prime}}\right\}$, where we use that $\operatorname{ker} \pi$ is smooth and finite. So in fact our map $\varphi$ factors over $M_{R^{\prime}}^{\circ} \rightarrow\left\{e_{R^{\prime}}\right\}$. This implies that $\varphi(m)=e_{R^{\prime}}$, as required.

For the statement about all centralizers we just note that $\pi \pi^{-1} H^{\prime}=H^{\prime}$ for all closed subgroup schemes $H^{\prime} \subseteq G^{\prime}$.

We are now in a position to prove Theorem 3.3 .

Proof of Theorem 3.3. According to (2.1) and Proposition 2.9, we can assume that $k=\bar{k}$ is algebraically closed. Let $H$ be any closed subgroup scheme of $G$. Using the following idea of [MT09, 2.8.1], we can first reduce to the case that $G$ is connected: Let $H$ act via conjugation on $G$. Since $G^{\circ}$ is normal in $G$, this induces an action of $H$ on $G^{\circ}$ and $\left(G^{\circ}\right)^{H}$ is a normal subgroup of $C_{G}(H)$. This allows us to consider the exact sequence of affine group schemes

$$
1 \rightarrow\left(G^{\circ}\right)^{H} \rightarrow C_{G}(H) \rightarrow C_{G}(H) /\left(G^{\circ}\right)^{H} \rightarrow 1 .
$$

It now remains to show that the left and right terms of this sequence are smooth (see Section 2.2). For the right-hand side, note that the closed embedding $C_{G}(H) \rightarrow G$ together with the fact that $C_{G}(H) \cap G^{\circ}=\left(G^{\circ}\right)^{H}$ induces a closed embedding $C_{G}(H) /\left(G^{\circ}\right)^{H} \rightarrow G / G^{\circ}$. Since $G / G^{\circ}$ is an étale group scheme (smooth and finite), the same is true for $C_{G}(H) /\left(G^{\circ}\right)^{H}$ (see Wat79, 6.2]). For the left-hand side, we claim that $\left(G^{\circ}\right)^{H}=\left(C_{G^{\circ}}\left(H \cap G^{\circ}\right)\right)^{\Gamma}$, where $\Gamma=H /\left(H \cap G^{\circ}\right)$. Indeed, $H$ acts via conjugation on $C_{G^{\circ}}\left(H \cap G^{\circ}\right)$ with fixed points $\left(G^{\circ}\right)^{H}$. But clearly, $H \cap G^{\circ}$ acts trivially, so the action factors through $\Gamma$ and we get the asserted equality. Now, as a closed subgroup scheme of $\pi_{0} G, \Gamma$ is again a smooth finite group scheme with order invertible in $k$, and hence linearly reductive. We can use Theorem 3.2 to conclude that the fixed points are again a smooth group scheme, provided that $C_{G^{\circ}}\left(H \cap G^{\circ}\right)$ is smooth. This allows us to assume from the outset that $G$ is connected and, by hypothesis, that the characteristic of $k$ is zero or pretty good for $G$.

If the characteristic is zero, all closed subgroup schemes are smooth. So let us assume that $p=\operatorname{char}(k)$ is a pretty good prime for $G$.

Let $G_{1}, \ldots, G_{t}$ be the simple components of the derived group of $G$. We may assume that $p$ is not very good for the root systems of $G_{1}, \ldots, G_{n}$, whereas it is for $G_{n+1}, \ldots, G_{t}$. Since $p$ is good for $G$ and hence for all components of the 
derived group, this implies that all groups $G_{1}, \ldots, G_{n}$ are of type $A$. Let $T$ be the radical of $G$ (which is a torus). Then multiplication in $G$ gives a central isogeny $T \times G_{1} \times \cdots \times G_{t} \rightarrow G$. Denote by $\tilde{G}$ the image of $T \times G_{1} \times \cdots \times G_{n}$ in $G$. Let $\pi: \tilde{G} \times G_{n+1} \times \cdots \times G_{t} \rightarrow G$ be the induced central isogeny. Due to our characteristic assumption, the Lie algebras Lie $\left(G_{i}\right), i>n$, are simple (see [Ho82, Cor. 2.7]). Using this, one can show that $\operatorname{Lie}(\operatorname{ker} \pi)=\operatorname{ker}(d \pi)=0$, i.e. that $\pi$ is separable. So, by Lemma 3.8, we may assume that $G=\tilde{G} \times G_{n+1} \times \cdots \times G_{t}$.

Now let $H \subseteq G$ be a closed subgroup scheme. Then we find that

$$
C_{G}(H)=C_{\tilde{G}}(\tilde{H}) \times C_{G_{n+1}}\left(H_{n+1}\right) \times \cdots \times C_{G_{t}}\left(H_{t}\right),
$$

where $H_{i}=p_{i} H$ for the projection $p_{i}: G \rightarrow G_{i}$, and similarly for $\tilde{H}$.

We claim that the centralizers $C_{G_{i}}\left(H_{i}\right), i>n$, are smooth. In fact, since every central isogeny between simple algebraic groups with the same root system in very good characteristic is separable, we need to prove the assertion only for one algebraic simple group with a given root system in very good characteristic. According to [Ri67], for a given root system and an algebraically closed field in very good characteristic, we can find a simple algebraic group $G_{i}^{\prime}$ of this type that fits into a reductive pair $\left(\mathrm{GL}_{m}, G_{i}^{\prime}\right)$ for a suitable $m$. We may conclude that the $C_{G_{i}}\left(H_{i}\right)$, $i>n$, are smooth by employing Lemmas 3.5, 3.6 and 3.8.

It remains to show that centralizers in $\tilde{G}$ are smooth. By Lemma 2.12 (d) and (e), $p$ is pretty good for $\tilde{G}$. By construction of $\tilde{G}$, and by replacing $G$ by $\tilde{G}$, we may assume that $G$ is a connected reductive group with root system $\Phi=A_{m_{1}} \times \cdots \times A_{m_{n}}$, where $p$ divides all integers $m_{1}+1, \ldots, m_{n}+1$. We also assume that the radical of $G$ has at least dimension $n$ (otherwise we replace $G$ by $G \times S$, for some torus $S$, and exploit the fact that $\left.C_{G \times S}(H \times 1)=C_{G}(H) \times S\right)$.

Let us now consider the reductive group $G_{\mathbb{C}}$ over $\mathbb{C}$ defined by the root datum $\left(X, \Phi, Y, \Phi^{\vee}\right)$ of $G$. We identify $G_{\mathbb{C}}$ and all other (smooth) complex algebraic groups below with their groups of complex points. The prime $p$ now is just a fixed prime that is pretty good for the root datum of $G_{\mathbb{C}}$; all we need is that it does not divide the order of the finite groups $Z\left(G_{\mathbb{C}}\right) / \operatorname{Rad}\left(G_{\mathbb{C}}\right)$ and $Z\left(G_{\mathbb{C}}^{\vee}\right) / \operatorname{Rad}\left(G_{\mathbb{C}}^{\vee}\right)$, which follows from the fact that the abelian groups $X / \mathbb{Z} \Phi$ and $Y / \mathbb{Z} \Phi^{\vee}$ have no $p$-torsion.

Let $r$ denote the dimension of the radical of $G_{\mathbb{C}}$. By assumption, $r \geq n$. To ease notation we set $\mathrm{SL}=\mathrm{SL}_{m_{1}+1} \times \cdots \times \mathrm{SL}_{m_{n}+1}, \mathrm{PSL}=\mathrm{PSL}_{m_{1}+1} \times \cdots \times \mathrm{PSL}_{m_{n}+1}$ and $\mathrm{GL}=\mathrm{GL}_{m_{1}+1} \times \cdots \times \mathrm{GL}_{m_{n}+1}$, considered as complex algebraic groups. We now fix some data that determines $G_{\mathbb{C}}$ : it is determined by its $\operatorname{subgroups} \operatorname{Der}\left(G_{\mathbb{C}}\right)$, $\operatorname{Rad}\left(G_{\mathbb{C}}\right)$ and the central isogeny $\operatorname{Der}\left(G_{\mathbb{C}}\right) \times \operatorname{Rad}\left(G_{\mathbb{C}}\right) \rightarrow G_{\mathbb{C}}$ given by multiplication. In our setup this means that there are normal, central, finite algebraic subgroups $M \subseteq N \subseteq \mathrm{SL}$ of SL and there is an injective homomorphism

$$
\varphi: N / M \rightarrow \mathbb{G}_{m}^{r}
$$

such that $G_{\mathbb{C}}$ is (isomorphic to) the quotient of

$$
N / M \rightarrow(\mathrm{SL} / M) \times \mathbb{G}_{m}^{r}, \quad a M \mapsto\left(a M, \varphi(a M)^{-1}\right) .
$$

Note that $M$ is the kernel of the natural isogeny $\mathrm{SL} \rightarrow \operatorname{Der}\left(G_{\mathbb{C}}\right)$ so that $\mathrm{SL} / M \cong$ $\operatorname{Der}\left(G_{\mathbb{C}}\right)$ and $N / M \cong \operatorname{Der}\left(G_{\mathbb{C}}\right) \cap \operatorname{Rad}\left(G_{\mathbb{C}}\right)$.

Let $Z=Z(\mathrm{SL})$. We claim that $p$ does not divide the orders of $M$ and $Z / N$. Indeed, the order of $M=\operatorname{ker}(\mathrm{SL} \rightarrow \mathrm{SL} / M)$ is the same as the order of $\operatorname{ker}\left((\mathrm{SL} / M)^{\vee}\right.$ 
$\rightarrow$ PSL), i.e. of $Z\left(\operatorname{Der}\left(G_{\mathbb{C}}\right)^{\vee}\right)=Z\left(G_{\mathbb{C}}^{\vee}\right) / \operatorname{Rad}\left(G_{\mathbb{C}}^{\vee}\right)$. On the other hand,

$$
\begin{aligned}
Z / N & \cong(Z / M) /(N / M) \cong Z\left(\operatorname{Der}\left(G_{\mathbb{C}}\right)\right) /\left(\operatorname{Rad}\left(G_{\mathbb{C}}\right) \cap \operatorname{Der}\left(G_{\mathbb{C}}\right)\right) \\
& \cong\left(Z\left(G_{\mathbb{C}}\right) \cap \operatorname{Der}\left(G_{\mathbb{C}}\right)\right) /\left(\operatorname{Rad}\left(G_{\mathbb{C}}\right) \cap \operatorname{Der}\left(G_{\mathbb{C}}\right)\right) \cong Z\left(G_{\mathbb{C}}\right) / \operatorname{Rad}\left(G_{\mathbb{C}}\right) .
\end{aligned}
$$

The assertion on $p$ follows.

Let $N_{p}$ denote the (unique) $p$-Sylow subgroup of $N$, and similarly $Z_{p} \subseteq Z$. The last paragraph implies that the composite $i: N_{p} \rightarrow N \rightarrow N / M$ is injective. It also implies that $N_{p}=Z_{p}$. Since $Z \cong \mu_{m_{1}+1} \times \cdots \times \mu_{m_{n}+1}$, this gives $N_{p} \cong$ $\mu_{p^{s_{1}}} \times \cdots \times \mu_{p^{s_{n}}}$, where $m_{i}+1=p^{s_{i}} m_{i}^{\prime}$ with $m_{i}^{\prime}$ and $p$ coprime. In particular, the inclusion $N_{p} \rightarrow Z$ corresponds to the standard inclusion $\mu_{p^{s_{1}}} \times \cdots \times \mu_{p^{s_{n}}} \rightarrow$ $\mu_{m_{1}+1} \times \cdots \times \mu_{m_{n}+1}$. We embed $Z$ into $\mathbb{G}_{m}^{n}$ via the componentwise inclusion of $\mu_{m_{1}+1} \times \cdots \times \mu_{m_{n}+1}$ into $\mathbb{G}_{m}^{n}$.

The injective homomorphism $\varphi \circ i: N_{p} \rightarrow \mathbb{G}_{m}^{r}$ yields a $\mathbb{Z}$-linear surjection of character groups $\mathbb{Z}^{r} \rightarrow \mathbb{Z} / p^{s_{1}} \times \cdots \times \mathbb{Z} / p^{s_{n}}$. Choose a matrix $A \in \mathbb{Z}^{n \times r}$ such that the above surjection is induced by

$$
\mathbb{Z}^{r} \stackrel{A}{\rightarrow} \mathbb{Z}^{n} \rightarrow \mathbb{Z} / p^{s_{1}} \times \cdots \times \mathbb{Z} / p^{s_{n}}
$$

in the obvious way. Since this concatenation is surjective, and since all $s_{i}$ are positive by assumption, the reduction of $A \bmod p$ is a surjective linear map $\mathbb{F}_{p}^{r} \rightarrow \mathbb{F}_{p}^{n}$ of $\mathbb{F}_{p}$-vector spaces. In particular, the elementary divisors $\alpha_{1}, \ldots, \alpha_{n}$ of $A$ over $\mathbb{Z}$ must all be prime to $p$. Up to change of basis on both sides over $\mathbb{Z}, A$ decomposes as a linear map as $\mathbb{Z}^{r} \rightarrow \mathbb{Z}^{n} \rightarrow \mathbb{Z}^{n}$, where the first map is the projection onto the first $n$ components, and the second map sends $\left(x_{1}, \ldots, x_{n}\right)$ to $\left(\alpha_{1} x_{1}, \ldots, \alpha_{n} x_{n}\right)$. Switching back to the category of multiplicative algebraic groups, we construct the following commutative diagram:

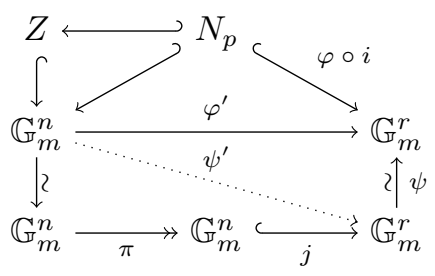

Here, $\varphi^{\prime}$ is the map induced by $A$, the isomorphisms correspond to the changes of bases, $j$ is the inclusion into the first $n$ coordinates of $\mathbb{G}_{m}^{r}$, and $\pi$ is a surjection with finite kernel of order prime to $p$ (this is due to the analysis of the elementary divisors above). In particular, the order of $\operatorname{ker}\left(\psi^{\prime}\right) \cong \operatorname{ker}(\pi)$ is prime to $p$.

We finally consider the following diagram with exact rows, defining complex connected reductive groups $H_{1}$ and $H_{2}$ :

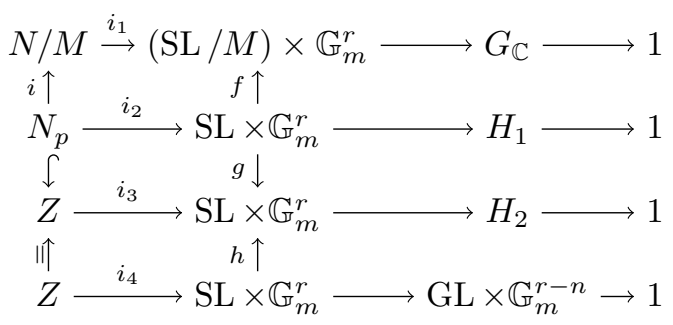


In this diagram, $i_{1}(a M)=\left(a M, \varphi(a M)^{-1}\right), i_{2}(a)=\left(a, \varphi^{\prime}(a)^{-1}\right), i_{3}(a)=\left(a, \varphi^{\prime}(a)^{-1}\right)$, $i_{4}(a)=\left(a, j(a)^{-1}\right)$; and $f(x, t)=(x M, t), g(x, t)=(x, t), h(x, t)=\left(x, \varphi^{\prime}\left(t_{1}, \ldots, t_{n}\right)\right.$ $\left.\psi\left(1, \ldots, 1, t_{n+1}, \ldots, t_{r}\right)\right)$. Per construction, all these maps are homomorphisms. The commutativity of (3.9) yields the commutativity of diagram (3.10), and moreover the surjectivity of $h$. Since $f$ and $g$ are also surjective, these maps induce surjective homomorphisms $\bar{f}: H_{1} \rightarrow G_{\mathbb{C}}, \bar{g}: H_{1} \rightarrow H_{2}$ and $\bar{h}: \mathrm{GL} \times \mathbb{G}_{m}^{r-n} \rightarrow H_{2}$. For $(x, t) \in \mathrm{SL} \times \mathbb{G}_{m}^{r}$ let us write $\overline{(x, t)}$ for the image of $(x, t)$ in $H_{1}$, and also (with slight abuse of notation) for its image in $\mathrm{GL} \times \mathbb{G}_{m}^{r-n}$. Using the definitions, we can verify that the maps

$$
\begin{aligned}
& \operatorname{ker}(\bar{f}) \rightarrow N / N_{p}, \overline{(x, t)} \mapsto x N_{p}, \\
& \operatorname{ker}(\bar{g}) \rightarrow Z / N_{p}, \overline{(x, t)} \mapsto x N_{p}, \\
& \operatorname{ker}(\bar{h}) \rightarrow \operatorname{ker}\left(\psi^{\prime}\right), \overline{(x, t)} \mapsto x\left(t_{1}, \ldots, t_{n}\right)
\end{aligned}
$$

are indeed well-defined inclusions of algebraic groups. In particular, $\bar{f}, \bar{g}$ and $\bar{h}$ are isogenies with kernels of order prime to $p$.

To conclude, we express the diagram of connected reductive groups and central isogenies

$$
G_{\mathbb{C}} \longleftarrow H_{1} \longrightarrow H_{2} \longleftarrow \mathrm{GL} \times \mathbb{G}_{m}^{r-n}
$$

in root data terms. This gives rise to connected reductive groups over $k$ and separable isogenies

$$
G \longleftarrow H_{1}^{\prime} \longrightarrow H_{2}^{\prime} \longleftarrow \mathrm{GL} \times \mathbb{G}_{m}^{r-n}
$$

We finish the proof by again referring to Lemmas 3.5 and 3.8

\section{Non-SMOOTH CENTRALIZERS}

In this section, let $k$ be an algebraically closed field of characteristic $p>0$ and let $G$ be a connected reductive algebraic group over $k$. Let $T$ be a maximal torus of $G$, with corresponding root datum $\left(X, \Phi, Y, \Phi^{\vee}\right)$.

Our method to construct examples of closed subgroup schemes of $G$ with nonsmooth centralizers rests on the following easy observation.

Lemma 4.1. Suppose $H$ is a closed subgroup scheme of $G$ containing $T$. Then $C=C_{G}(H)=Z(H)$ is diagonalizable. In particular, it is smooth if and only if the p-torsion of its character group $X(C)$ vanishes.

Proof. We have $C=C_{G}(H) \subseteq C_{G}(T)=T \subseteq H$, so $C$ is diagonalizable and coincides with the center of $H$. (For the smoothness criterion see DG70, IV, $§ 1$, $1.2]$.

Example 4.2. (a) Suppose $p$ is a bad prime for $G$. Then, Lemmas 2.10 and 4.1 suggest how to find a subgroup $H$ such that $C_{G}(H)$ is not smooth:

According to Lemma 2.10, we can pick a subset $\Phi^{\prime} \subseteq \Phi$ such that the $p$-torsion of $\mathbb{Z} \Phi / \mathbb{Z} \Phi^{\prime}$ does not vanish. We may assume that $\overline{\Phi^{\prime}}$ is closed and symmetric (otherwise we replace it by $\mathbb{Z} \Phi^{\prime} \cap \Phi$ ). Now the connected reductive group $H$ generated by $T$ together with the root groups $U_{\alpha}, \alpha \in \Phi^{\prime}$, has a center with character group $X(T) / \mathbb{Z} \Phi^{\prime}$ (see [Ja03, II, 1.6 and 1.7]), which contains $\mathbb{Z} \Phi / \mathbb{Z} \Phi^{\prime}$. So $C_{G}(H)$ is non-smooth, by Lemma 4.1 
Since we did not give a proof of Lemma 2.10, it may be convenient to now explicitly describe how to obtain a closed symmetric subset $\Phi^{\prime}$ as above. By definition of a bad prime, we can find an irreducible component $\Phi_{1}$ of $\Phi$ such that $p$ divides a coefficient of the highest root $\beta$ of $\Phi_{1}$ relative to some base $\Delta_{1}=\left\{\alpha_{1}, \ldots, \alpha_{r}\right\}$ of $\Phi_{1}$. Suppose that the coefficient $m_{1}$ of $\alpha_{1}$ in $\beta$ is divisible by $p$. Let $\Delta$ be a base of $\Phi$ containing $\Delta_{1}$. Let $\Phi^{\prime}$ be the subroot system with base $\Delta^{\prime}=\left(\Delta \backslash\left\{\alpha_{1}\right\}\right) \cup\{-\beta\}$. Now the $p$-torsion of $\mathbb{Z} \Phi / \mathbb{Z} \Phi^{\prime}$ equals $\mathbb{Z} / p^{r} \mathbb{Z}$, where $p^{r}$ is the $p$-part of the integer $m_{1}$. Note that in the language of the Borel-de Siebenthal algorithm (see e.g. Go07, $2.1]$ ), we have obtained $\Phi^{\prime}$ (and hence $H$ ) by "crossing out" the node $\alpha_{1}$ in the extended Dynkin diagram corresponding to $\Delta_{1}$, i.e. by crossing out a single node with a coefficient that is divisible by $p$.

(b) Suppose that $G$ has root system $\Phi=A_{m_{1}} \times \cdots \times A_{m_{n}}$ and that $p$ is not pretty good for $G$. Again, we want to find a subgroup $H$ such that $C_{G}(H)$ is not smooth.

If the center $Z(G)$ is not smooth, we are done. Due to Lemma 2.12 (a) (and because all primes are good for $\Phi)$ this means that we can assume that $Y / \mathbb{Z} \Phi^{\vee}$ has $p$-torsion. Let $s=s_{1} \cdots s_{n}$ be the element of the Weyl group of $G$ obtained by multiplying Coxeter elements $s_{i}$ of the Weyl groups of type $A_{m_{i}}$. Here we take $s_{i}=s_{i 1} \cdots s_{i m_{i}}$, where the $s_{i j}$ are the simple reflections for some chosen root base $\Delta_{i}=\left\{\alpha_{i 1}, \ldots, \alpha_{i m_{i}}\right\}$ of $A_{m_{i}}$, which is ordered as in Bou68, Planche I]. Then $\Delta=\Delta_{1} \cup \cdots \cup \Delta_{n}$ is a root base of $\Phi$, and hence a basis of $\mathbb{Z} \Phi$. Let $\Delta^{\vee}$ be the corresponding dual base of $\Phi^{\vee}$. For $\lambda \in X$ an easy induction on $n$ (and for $n=1$ on $m_{1}$ ) proves the equation

$$
s(\lambda)=\lambda-\sum_{i=1}^{n} \sum_{j=1}^{m_{i}} \sum_{k=j}^{m_{i}}\left\langle\lambda, \alpha_{i k}^{\vee}\right\rangle \alpha_{i j} .
$$

Consider the map $s-1: X \rightarrow \mathbb{Z} \Phi, \lambda \mapsto s(\lambda)-\lambda$. We are going to compute the elementary divisors of $(s-1) X$ in $\mathbb{Z} \Phi$. Let $\mathfrak{b}=\left\{\lambda_{1}, \ldots, \lambda_{r}\right\}$ be a basis of $X$ and $\mathfrak{b}^{\vee}=\left\{\lambda_{1}^{\vee}, \ldots, \lambda_{r}^{\vee}\right\}$ a dual basis of $Y$ with respect to $\langle$,$\rangle . Then the matrix M$ of $(s-1)$ with respect to $\mathfrak{b}$ and $\Delta$ is given by the entries

$$
M_{(i, j), l}=-\sum_{k=j}^{m_{i}}\left\langle\lambda_{l}, \alpha_{i k}^{\vee}\right\rangle,
$$

where $(i, j)$ varies in $i=1, \ldots, n, j=1, \ldots, m_{i}$, and $l=1, \ldots, r$. We have to compute the elementary divisors of $M$. We may replace each $(i, j)$-th row by the $(i, j+1)$-th row minus the $(i, j)$-th row, for $i=1, \ldots, n$ and $j<m_{i}$, and obtain a matrix with entries $\left\langle\lambda_{l}, \alpha_{i j}^{\vee}\right\rangle$. But this is precisely the transpose of the matrix describing the map $\mathbb{Z} \Phi^{\vee} \hookrightarrow Y$ with respect to the bases $\Delta^{\vee}$ and $\mathfrak{b}^{\vee}$. We conclude that the elementary divisors of $(s-1) X$ in $\mathbb{Z} \Phi$ coincide with the elementary divisors of $\mathbb{Z} \Phi^{\vee}$ in $Y$ so that the group $\mathbb{Z} \Phi /(s-1) X$ has $p$-torsion. Hence also the group $X /(s-1) X$ has $p$-torsion. This is the character group of $T^{\sigma}$, where $\sigma=s^{-1}$. Hence $T^{\sigma}$ is a non-smooth group scheme by the smoothness criterion referred to in the proof of Lemma 4.1. For a chosen representative $n_{\sigma} \in N_{G}(T)$, we may write this group as $C_{G}(H)$, where $H$ is the subgroup generated by $T$ and $n_{\sigma}$. Our search has come to an end. 
Combined with Theorem 3.3, these examples allow us to prove the theorem from the introduction:

Proof of Theorem 1.1. The forward implication is clear by Theorem 3.3. Now suppose all centralizers in $G$ are smooth. According to Example 4.2 (a), the characteristic needs to be good. Under this assumption we showed in the proof of Theorem 3.3 that there is a separable isogeny $\tilde{G} \times H \rightarrow G$, where $H$ is defined in very good characteristic, and $\tilde{G}$ is as in Example 4.2 (b). If the characteristic was not pretty good for $\tilde{G}$, Example 4.2 (b) would provide a subgroup scheme $\tilde{H}$ such that $C_{\tilde{G} \times H}(\tilde{H} \times 1)=C_{\tilde{G}}(\tilde{H}) \times H$ would be non-smooth. By Lemma 3.8, this would give rise to a non-smooth centralizer in $G$, contradicting our assumption. So the characteristic must be pretty good for $\tilde{G}$, and hence for $\tilde{G} \times H$, by Lemma 2.12 (e). According to part (d) of the same lemma, it is also pretty good for $G$.

Remark 4.3. Theorem 1.1 also shows that the existence of a reductive pair $\left(\mathrm{GL}_{n}, G\right)$ (for some $n$ ) puts restrictions on the characteristic of $k$. Indeed, suppose that the characteristic is not pretty good for $G$. Then the existence of non-smooth centralizers implies (according to Lemmas 3.5 and 3.6) that we can never find an inclusion $G \rightarrow \mathrm{GL}_{n}$ that gives a reductive pair $\left(\mathrm{GL}_{n}, G\right)$.

Remark 4.4. The groups $H$ in Example 4.2 (a) and (b) have in common that they are smooth, reductive (not necessarily connected), and contain a maximal torus of $G$. Using Lemma 3.1 and Lemma 3.8, the proof of Theorem 1.1 can be strengthened to yield the equivalence of the following three statements about a connected reductive group $G$ :

(a) $\operatorname{char}(k)$ is zero or pretty good for $G$,

(b) all centralizers of closed subgroups schemes of $G$ are smooth,

(c) all (smooth) reductive subgroups of $G$ that contain a maximal torus are separable in $G$.

\section{Standard Reductive Groups}

We finally want to relate our notion of pretty good primes to existing notions of so-called standard reductive groups. Let $k$ be an algebraically closed field. Consider the following conditions on a connected reductive group $G$ over $k$ :

- $G$ is standard, i.e. the derived group of $G$ is simply connected, $\operatorname{char}(k)$ is good for $G$, and $\operatorname{Lie}(G)$ carries a non-degenerate bilinear form that is invariant under the $G$-action (these are the "standard hypotheses" of Jantzen; see [Ja04, 2.9]).

- $G$ is $T$-standard, i.e. $G$ is of the following form: it is separably isogenous to a Levi subgroup of $H$, where $H$ is a smooth group scheme of the form $H=H_{1} \times T$ with a torus $T$ and a semisimple group $H_{1}$ for which the characteristic of $k$ is very good (this notion is due to McNinch; see McN08; they are also called "strongly standard" in [McN05]).

- $G$ is $D$-standard, i.e. it is separably isogenous to a group $C_{H}(D)^{\circ}$, where $H$ is as in the case above, and where $D \subseteq H$ is a (not necessarily smooth) subgroup scheme of multiplicative type (this notion is due to McNinch and Testerman; see MT09; for smooth $D$ they are called "strongly standard" in MT07]). We note here that, in fact, a group of the form $C_{H}(D)^{\circ}$ is 
always reductive: it is smooth due to Theorem 3.2 and $H / C_{H}(D)$ is affine ([SGA3, XII, 5.5]), which implies reductivity ([Ri77]).

We slightly enlarge the classes of connected reductive groups defined by these properties: We say that $G$ is essentially standard (resp. essentially T-standard, essentially D-standard) if it may be obtained from a standard (resp. T-standard, $D$-standard) group $H$ after a finite number of the following operations:

(i) the replacement of $H$ by a separably isogenous group;

(ii) the replacement of $H=H^{\prime} \times S$ by $H^{\prime}$, where $S$ is a torus.

Remark 5.1. Cancelling off a torus factor really has some effect. For example, consider the groups $G=\mathrm{GL}_{5}$ and $G^{\prime}=\mathrm{GL}_{5} / \mu_{2}$, where $\mu_{2}$ is embedded in the center in the obvious way. Then $G$ and $G^{\prime}$ are non-isomorphic connected reductive groups. However, the groups $G \times \mathbb{G}_{m}$ and $G^{\prime} \times \mathbb{G}_{m}$ are isomorphic. This can be verified by a small root data calculation. It implies that operation (ii) applied to $G \times \mathbb{G}_{m}$ may produce $G^{\prime}$.

Theorem 5.2. Let $G$ be a connected reductive group defined over $k$. Then the following are equivalent:

(a) The characteristic of $k$ is zero or pretty good for $G$,

(b) $G$ is essentially standard,

(c) $G$ is essentially $T$-standard,

(d) $G$ is essentially D-standard.

Proof. Any Levi subgroup of a connected reductive group may be written as the centralizer of a suitable torus. So clearly (c) implies (d).

Suppose (d) is satisfied. We claim that (a) holds. According to Theorem 1.1 it suffices to show that all centralizers in $G$ are smooth. By Lemma 3.8 we may assume that $G=C_{H}(D)^{\circ}$, where $D \subseteq H$ is a diagonalizable subgroup scheme, and where $H$ is a reductive group in very good characteristic. It now suffices to show, by Theorem 3.3 and Lemma 3.6. that $\left(H, C_{H}(D)^{\circ}\right)$ is a reductive pair. We can finish the argument by employing the ideas of [BMRT, Prop. 3.7 (a)]: As a $D$-module, the Lie algebra $\mathfrak{h}=\operatorname{Lie}(H)$ decomposes into a direct sum $\mathfrak{h}=\bigoplus_{\chi} \mathfrak{h}_{\chi}$, where $\chi$ varies over the character group of $D$ (see [DG70, II, §2, 2.5]). Moreover, $\mathfrak{h}_{0}=\mathfrak{h}^{D}=\operatorname{Lie}\left(C_{H}(D)\right)=\operatorname{Lie}\left(C_{H}(D)^{\circ}\right)$ so that the reductive pair condition is satisfied.

Suppose that (a) holds. In the proof of Theorem 3.3 we have shown that up to separable isogenies and direct products with a torus, $G$ is a direct product of groups of the following form: a torus, some $\mathrm{GL}_{n}$, and a simple group in very good characteristic. Since $\mathrm{GL}_{n}$ is $T$-standard (see e.g. [McN05, Rem. 3]), the whole direct product is $T$-standard, and (c) holds.

Suppose $G$ is $T$-standard. Then $G$ is separably isogenous to a standard group, by [McN05, Prop. 2]. This means that (c) implies (b).

Suppose that $G$ is standard. Then the center $Z(G)$ is smooth (by the argument of [MT09, 3.4.2]: $Z(G)=C_{G}(X)^{\lambda}$ for some $X \in \operatorname{Lie}(G)$ and some cocharacter $\lambda$, and Jantzen's conditions guarantee that the centralizer $C_{G}(X)$ is smooth). The same is true for $Z\left(G^{\vee}\right)$, for example, since $Z\left(G^{\vee}\right) / \operatorname{Rad}\left(G^{\vee}\right)$ is trivial due to the fact that $\operatorname{Der}(G)$ is simply connected. Since $p$ is also good, it is pretty good, by Lemma 2.12 (a). Since the class of groups in pretty good characteristic is closed under the operations (i) and (ii) above, any essentially standard group is now defined in 
pretty good characteristic. We have shown that (b) implies (a), which finishes the proof.

By Theorem 1.1, we deduce the following consequence.

Corollary 5.3. Let $G$ be a connected reductive group satisfying any of the three standardness conditions above. Then all centralizers of closed subgroup schemes in $G$ are smooth.

Remark 5.4. George McNinch suggested that we give an easier direct description of essentially standard groups, starting from the easiest examples, and operations such as (i) and (ii) above. We can do this as follows. Consider the class of connected reductive groups with the following properties:

- it contains all simple groups defined in very good characteristic;

- it contains $\mathrm{GL}_{n}$ for all $n$;

- it contains all tori;

- it is closed under taking products and operations (i), (ii) as above.

By Lemma 2.12, all groups in this class are defined in pretty good characteristic (for $\mathrm{GL}_{n}$ we use part (a) of the lemma and the fact that in this case $X(T) / \mathbb{Z} \Phi \cong$ $\left.\mathbb{Z} \cong Y(T) / \mathbb{Z} \Phi^{\vee}\right)$. The converse follows from the proof of the implication (a) $\Rightarrow$ (c) in Theorem 5.2 above.

By the characterization in terms of the smoothness of all centralizers, the class of groups defined in pretty good characteristic is closed under taking subgroups such that we obtain a reductive pair. In particular, it is closed under taking centralizers of diagonalizable subgroup schemes (cf. the proof of $(d) \Rightarrow$ (a) in Theorem 5.2).

The last remark gives a very easy recipe to prove results for groups defined in pretty good characteristic. We illustrate this with the existence of Springer isomorphisms.

Corollary 5.5. Let $G$ be a connected reductive group defined in pretty good characteristic. Then there is a $G$-equivariant isomorphism of varieties $\mathcal{U} \rightarrow \mathcal{N}$, where $\mathcal{U} \subseteq G$ (resp. $\mathcal{N} \subseteq \mathfrak{g}$ ) is the unipotent (resp. nilpotent) variety associated to $G$.

Proof. The result holds for $\mathrm{GL}_{n}$ and for tori. In [McN05, Prop. 9], McNinch shows how to extend Springer's work to $T$-standard groups by first extending the result to semisimple groups defined in very good characteristic. He also shows that the existence of Springer isomorphisms is compatible with operation (i). The compatibility with operation (ii) and with taking direct products is obvious.

\section{ACKNOWLEDGEMENTS}

This paper was prepared towards the author's Ph.D. qualification under the supervision of Gerhard Röhrle, with financial support of the DFG-priority program SPP1388 "Representation Theory". The author thanks Gerhard Röhrle for help in preparing this paper. He is also very grateful to Olivier Brunat for helpful discussions and to Steve Donkin for pointing out the example in Remark 3.4 (c). The author is indebted to George McNinch for comments and questions on an earlier version of this manuscript, and thanks the referee for numerous improvements of the exposition. Finally the author acknowledges additional support from ERC Advanced Grant 291512. 


\section{REFERENCES}

[BMR05] M. Bate, B. Martin, and G. Röhrle. A geometric approach to complete reducibility. Invent. Math., 161(1):177-218, 2005. MR2178661 (2007k:20101)

[BMRT] M. Bate, B. Martin, G. Röhrle, and R. Tange. Complete reducibility and separability. Trans. Amer. Math. Soc., 362(8):4283-4311, 2010. MR2608407 (2011i:20068)

[Bo91] A. Borel. Linear algebraic groups, volume 126 of Graduate Texts in Mathematics. Springer-Verlag, New York, second edition, 1991. MR.1102012 (92d:20001)

[Bou68] N. Bourbaki. Éléments de mathématique. Fasc. XXXIV. Groupes et algèbres de Lie. Chapitre IV: Groupes de Coxeter et systèmes de Tits. Chapitre V: Groupes engendrés par des réflexions. Chapitre VI: Systèmes de racines. Actualités Scientifiques et Industrielles, No. 1337, Hermann, Paris, 1968. MR0240238 (39:1590)

[DG70] M. Demazure and P. Gabriel. Groupes algébriques. Tome I: Géométrie algébrique, généralités, groupes commutatifs. Masson \& Cie, Éditeur, Paris, 1970. Avec un appendice sur Corps de classes local par Michiel Hazewinkel. MR0302656 (46:1800)

[SGA3] M. Demazure and A. Grothendieck. Schémas en groupes, Séminaire de Géométrie Algébrique du Bois Marie 1962/64 (SGA 3). (Lecture Notes in Math., 151-153). Springer-Verlag, Berlin, 1970.

[Go07] S. M. Goodwin. On generation of the root lattice by roots. Math. Proc. Cambridge Philos. Soc., 142(1):41-45, 2007. MR2296389(2007m:17012)

[Ho82] G. M. D. Hogeweij. Almost-classical Lie algebras. I, II. Nederl. Akad. Wetensch. Indag. Math., 44(4):441-452, 453-460, 1982. MR683531 (84f:17007)

[Ja03] J. C. Jantzen. Representations of algebraic groups, volume 107 of Mathematical Surveys and Monographs. American Mathematical Society, Providence, RI, second edition, 2003. MR2015057 (2004h:20061)

[Ja04] J. C. Jantzen. Nilpotent orbits in representation theory. In Lie theory, volume 228 of Progr. Math., pages 1-211. Birkhäuser Boston, Boston, MA, 2004. MR2042689 (2005c:14055)

[KMRT] M-A. Knus, A. Merkurjev, M. Rost, and J-P. Tignol. The book of involutions, volume 44 of American Mathematical Society Colloquium Publications. American Mathematical Society, Providence, RI, 1998. With a preface in French by J. Tits. MR.1632779 (2000a:16031)

[LT99] R. Lawther and D. M. Testerman. A1 subgroups of exceptional algebraic groups. Mem. Amer. Math. Soc., 141(674):viii+131, 1999. MR1466951(2000b:20059)

[LS96] M. W. Liebeck and G. M. Seitz. Reductive subgroups of exceptional algebraic groups. Mem. Amer. Math. Soc., 121(580):vi+111, 1996. MR1329942 (96i:20059)

[Liu02] Q. Liu. Algebraic geometry and arithmetic curves, volume 6 of Oxford Graduate Texts in Mathematics. Oxford University Press, Oxford, 2002. Translated from the French by Reinie Erné, Oxford Science Publications. MR 1917232 (2003g:14001)

[McN05] G. J. McNinch. Optimal SL(2)-homomorphisms. Comment. Math. Helv., 80(2):391-426, 2005. MR2142248(2006f:20055)

[McN08] G. J. McNinch. The centralizer of a nilpotent section. Nagoya Math. J., 190:129-181, 2008. MR2423832 (2009d:20110)

[MT07] G. J. McNinch and D. M. Testerman. Completely reducible SL(2)-homomorphisms. Trans. Amer. Math. Soc., 359(9):4489-4510 (electronic), 2007. MR2309195 (2008d:20084)

[MT09] G. J. McNinch and D. M. Testerman. Nilpotent centralizers and Springer isomorphisms. J. Pure Appl. Algebra, 213(7):1346-1363, 2009. MR2497582 (2010c:20059)

[Ri67] R. W. Richardson. Conjugacy classes in Lie algebras and algebraic groups. Ann. of Math. (2), 86:1-15, 1967. MR0217079 (36:173)

[Ri77] R. W. Richardson. Affine coset spaces of reductive algebraic groups. Bull. London Math. Soc., 9(1):38-41, 1977. MR0437549 (55:10473)

[Sp98] T. A. Springer. Linear algebraic groups, volume 9 of Progress in Mathematics. Birkhäuser Boston, Inc., Boston, MA, second edition, 1998. MR1642713 (99h:20075)

[SpSt70] T. A. Springer and R. Steinberg. Conjugacy classes. In Seminar on Algebraic Groups and Related Finite Groups (The Institute for Advanced Study, Princeton, N.J., 1968/69), Lecture Notes in Mathematics, Vol. 131, pages 167-266. Springer, Berlin, 1970. MR0268192(42:3091) 
[Wat79] W. C. Waterhouse. Introduction to affine group schemes, volume 66 of Graduate Texts in Mathematics. Springer-Verlag, New York, 1979. MR547117 (82e:14003)

FAkultät Für Mathematik, Ruhr-Universität Bochum, 44780 Bochum, Germany

E-mail address: sebastian.herpel@rub.de

Current address: Fachbereich Mathematik, TU Kaiserslautern, Postfach 3049, 67653 Kaiserslautern, Germany 